\title{
Exponentially Accurate Error Estimates of Quasiclassical Eigenvalues
}

\author{
Julio H. Toloza
}

\begin{abstract}
Dissertation submitted to the Faculty of the
Virginia Polytechnic Institute and State University

in partial fulfillment of the requirements for the degree of
\end{abstract}

Doctor of Philosophy

in

Mathematical Physics

Dr. George Hagedorn, Chair

Dr. Lay Nam Chang

Dr. Martin Klaus

Dr. Beate Schmittmann

Dr. Werner Kohler

December 11, 2002

Blacksburg, Virginia

Keywords: Semiclassical Limit, Low-Lying Eigenvalues, Rayleigh-Schrödinger Perturbation Theory, Quasimodes

Copyright 2002, Julio H. Toloza 


\section{Exponentially Accurate Error Estimates of Quasiclassical Eigenvalues}

Julio H. Toloza

(ABSTRACT)

We study the behavior of truncated Rayleigh-Schrödinger series for the low-lying eigenvalues of the time-independent Schrödinger equation, in the semiclassical limit $\hbar \searrow 0$. Under certain hypotheses on the potential $V(x)$, we prove that for any given small $\hbar>0$ there is an optimal truncation of the series for the approximate eigenvalues, such that the difference between an approximate and actual eigenvalue is smaller than $\exp (-C / \hbar)$ for some positive constant $C$. We also prove the analogous results concerning the eigenfunctions. 


\section{Dedication}

To my mother. 


\section{Acknowledgments}

I am greatly indebted to my advisor Dr. George Hagedorn, who always has expressed confidence in my work. His intellectual guidance and support have been crucial to complete this dissertation.

I would like to thank Dr. Martin Klaus for his support to enrich and continue my academic career.

I am also thankful to my former advisor Dr. Guido Raggio who introduced me to the Mathematical Physics.

A very special thanks to Chris Thomas. My life as a graduate student has been much easier because of her ever timely help.

Finally, I want to express my gratitude to Natacha, for her patience, support, and understanding. 


\section{Contents}

1 Introduction $\quad 1$

2 Preliminaries $\quad 8$

2.1 R-S perturbation theory . . . . . . . . . . . . . . . 11

2.2 Some technical results . . . . . . . . . . . . . . . . . . . 15

3 Computation of the R-S coefficients $\quad 22$

3.1 Degeneracy is preserved. . . . . . . . . . . . . . . . 22

3.2 Degeneracy is removed. . . . . . . . . . . . . . . . . 27

$\begin{array}{lll} & \text { The main estimate } & 41\end{array}$

5 Optimal truncation $\quad 52$

6 Conclusion $\quad 64$ 
6.1 Relaxing the hypotheses . . . . . . . . . . . . . . . 65

6.2 Asymptotics on the Born-Oppenheimer approximation . . . . . . . . . 67

$\begin{array}{ll}\text { A Simplifying } \xi_{N}(x) & 69\end{array}$

$\begin{array}{ll}\text { Bibliography } & 71\end{array}$

$\begin{array}{ll}\text { Vita } & 75\end{array}$ 


\section{Chapter 1}

\section{Introduction}

Perhaps one of the most elementary facts in Quantum Physics is that, for a sufficiently deep potential well, the eigenvalue problem defined by the time-independent Schrödinger equation admits normalizable solutions. That is, one expects that there are at least one square-integrable function $\tilde{\Psi}(\hbar ; x)$ and a number $E(\hbar)$ that satisfy

$$
H(\hbar) \tilde{\Psi}(\hbar ; x):=\left[-\frac{\hbar^{2}}{2} \Delta_{x}+V(x)\right] \tilde{\Psi}(x)=E(\hbar) \tilde{\Psi}(\hbar ; x)
$$

provided that the potential energy has a "deep enough" global minimum. Equivalently, if one considers the Planck's constant as a parameter, then the equation above is expected to have solutions for small values of $\hbar>0$. Since one looks for solutions near the bottom of the potential well, this statement is often referred to as the existence of low-lying eigenvalues in the semiclassical limit $\hbar \searrow 0$.

Along with the problem of existence of low-lying eigenvalues, one is also interested in the 
behavior of the corresponding perturbation series in powers of $\hbar$, the so-called RayleighSchrödinger (R-S) series. It is well known that, in general, the R-S series are not convergent but only asymptotic to the solutions of equation (1.1). However, one often wants to consider truncations of these series as good approximations to the actual eigenvalues/eigenvectors. This raises the natural question of whether or not one can find an optimal truncation that minimizes the difference between the exact eigenvalues/eigenvectors and the corresponding truncated R-S series.

In this dissertation we aim to find exponentially accurate asymptotics to the solutions of (1.1). We shall prove that, under certain conditions of analyticity and growth on the potential energy, one can truncate the R-S series so that the difference between the truncated series and the actual eigenvalue/eigenvector can be made smaller than $\exp (-C / \hbar)$ for some positive constant $C>0$. Our construction is based entirely on a straighforward application of the RS perturbation theory, as opposed to the technically awkward quantization of normal forms. This latter technique is briefly described below. The results to be discussed here are already published in two papers $[27,28]$. The one-dimensional problem is considered in [27]. The multidimensional problem, which involves degenerate perturbation theory, is discussed in [28]. A review of results can be found in [29].

Rigorous results concerned with the discrete spectrum of (1.1), in the semiclassical limit $\hbar \searrow 0$, were missing until not long ago. The first proof of existence of low-lying eigenvalues and asymptotic R-S series was presented by Combes et al in 1983. Their proof, which involves Dirichlet-Newmann bracketing and the Krein's formula, only considers the one-dimensional 
problem. Shortly after, Simon gave another proof, based on geometric arguments, that is valid in several dimensions [24]. In both cases, the potential energy $V(x)$ is assumed to be a sufficiently smooth function which has several global minima, each one admiting a nonvanishing second order approximation. Those terms are separated from the potential energy term, and a suitable scaling is made on the Schrödinger equation. The quadratic pieces along with the kinetic energy term are treated as an unperturbed harmonic oscillator hamiltonian, and the remainder is considered as a perturbation of it. Then, for small values of $\hbar$ and near the bottom of $V(x)$, the whole hamiltonian is expected to be "close" to the harmonic oscillator and therefore its low-lying eigenvalues should be also close to those of the harmonic oscillator. Following the same underlying idea, but applying the so-called "twisting trick" [22, Section IX.11], Howland presented another proof for the one-dimensional problem [11]. Along with the existence of low-lying eigenvalues, the aforementioned results state that the low-lying eigenvalues of $H(\hbar)$ are given asymptotically by R-S series, in the sense that

$$
\left|E(\hbar)-\hbar \sum_{n=0}^{N} \mathcal{E}_{n} \hbar^{\frac{n}{2}}\right| \leq C_{N} \hbar^{\frac{N+1}{2}+1}
$$

for any given $N$, and sufficiently small $\hbar>0$. An analogous statement holds for the corresponding eigenvectors. We also must mention that this problem has been studied by Helffer and Sjöstrand in the framework of microlocal analysis (see below) [10].

The semiclassical limit of $H(\hbar)$ has also been studied from a rather different approach, namely, by quantization of the canonical perturbation theory. In this context, the potential energy is split in the same way as described above. However, one now first considers the perturbation problem of the classical hamiltonian and the properties of the associated 
perturbation series. Briefly speaking, one looks for approximate solutions to the canonical equations for a hamiltonian of the form

$$
h(A, \phi)=h_{0}(A)+\epsilon f(A, \phi),
$$

where $h_{0}(A)$ is the hamiltonian of a canonically integrable system (in our case, a harmonic oscillator), already expressed as a function of the canonically conjugated action-angle variables $A=\left(A_{1}, \ldots, A_{d}\right)$ and $\phi=\left(\phi_{1}, \ldots, \phi_{d}\right)$. The variables $A_{i}$ 's are essentially defined by the conserved quantities (for the harmonic oscillator, they are the energy contributions from each coordinate $x_{i}$ ). Each angle variable $\phi_{i}$ takes values in the unit circle $T$. It is clear that all the solutions to $h_{0}(A)$, with the same initial datum $A$, wind around the $d$-dimensional torus $A \times T^{d}$ with constant frequencies $\omega_{i}=\partial h(A) / \partial A_{i}$. The perturbation $f(A, \phi)$ is assumed to be bounded below and sufficiently smooth. Then the Kolmogorov-Arnold-Moser (KAM) theorem states that the solutions to the perturbed hamiltonian, for small $\epsilon$, also lie on invariant tori that are close to the invariant tori of $h_{0}(A)$, provided that $h_{0}(A)$ satisfies certain condition of non-degeneracy (see below). Moreover, for any given order $N$, one can construct a canonical map $(A, \phi) \rightarrow\left(A^{\prime}, \phi^{\prime}\right)$ such that (1.3) becomes

$$
h\left(A^{\prime}, \phi^{\prime}\right)=h_{\epsilon}^{(N)}\left(A^{\prime}\right)+\epsilon^{N+1} f_{\epsilon}^{(N)}\left(A^{\prime}, \phi^{\prime}\right)
$$

where $h_{\epsilon}^{(N)}\left(A^{\prime}\right)$ can be expressed in terms of the Birkhoff normal forms

$$
h_{\epsilon}^{(N)}\left(A^{\prime}\right)=h_{0}\left(A^{\prime}\right)+\sum_{n=1}^{N} \epsilon^{n} N_{0}^{(n)}\left(A^{\prime}\right) .
$$

The corpus of mathematical results on canonical perturbation theory, which describes geometrical features in phase-space, is known as KAM theory. See e. g., [1, 5, 15]. The 
quantization of the classical perturbation series is done afterward by resorting to several PDE techniques generically known as semiclassical (or microlocal) analysis. The literature on this subject is vast. See, for instance, $[10,6,16]$. This approach establishes a profound link between the classical problem and its quantum counterpart. Different aspects of the phase-space dynamics are in this way associated with spectral properties of the quantum system in the semiclassical limit. On the other hand, the KAM theorem imposes a restriction to this technique. As we have mentioned above, the KAM theorem is valid under a assumption of non-degeneracy. For a harmonic oscillator this means that the frequencies $\left(\omega_{1}, \ldots, \omega_{d}\right)$ must fulfill the non-resonance condition $\left|\sum_{i} \omega_{i} k_{i}\right|^{-1} \leq C\left(\sum_{i}\left|k_{i}\right|\right)^{\alpha}$, for $C>0, \alpha>0$, and for every non-trivial set of integers $\left(k_{1}, \ldots, k_{d}\right)$. Therefore, quantization of the KAM theory seems to be inadequate to handle hamiltonian operators whose harmonic oscillator part (after splitting of the potential energy term) fails to satisfy this latter condition.

The inequality (1.2) establishes an error estimate of the form $\mathcal{O}\left(\hbar^{N}\right)$. Assuming the nonresonance condition, more refined asymptotic formulas have been achieved by quantization of the KAM theory. Sjöstrand [26] obtained an asymptotic formula up to $\mathcal{O}\left(\hbar^{\infty}\right)$, valid for all the eigenvalues within the interval $\left[0, \hbar^{\delta}\right], \delta>0$. His construction is based on pseudodifferential functional calculus applied to the Birkhoff normal forms, where $V(x)$ is assumed to be $C^{\infty}$ with a single minimum. Sharper error estimates were proved by further assuming that the potential energy belonged to $G^{\mu}$, the collection of Gevrey class functions of order $\mu \geq 1$. Roughly speaking, $G^{\mu \geq 1}$ classes "interpolate smoothness" between $C^{\infty}$ and the set of analytic functions in a certain domain. The latter actually coincides with $G^{1}$. For a 
precise definition of Grevrey class see e. g., [16]. For $V(x) \in G^{\mu}$ with $\mu>1$, Bambusi et al [2] proposed another asymptotic formula that is valid in an energy interval of the form $\left[0,|\log \hbar|^{b}\right]$. Their quantization formula turns the error estimate $\mathcal{O}\left(\hbar^{\infty}\right)$ into $\mathcal{O}\left(\exp \left(-c / \hbar^{\beta}\right)\right)$ for the eigenvalues in the interval $\left[0, \hbar^{\delta}\right]$, where $0<\beta<1$ is related to both the order of the Gevrey class and the way that the non-resonance condition is satisfied. Quantization formulae valid for energies within an interval of the form $[0, M]$ have been stated by Popov $[19,20]$. These results also led to error terms of the form $\mathcal{O}\left(\exp \left(-c / \hbar^{\beta}\right)\right), 0<\beta<1$, for Gevrey class potential functions of order strictly bigger than one.

Although quantization of the KAM theory is a powerful technique for the investigation of the semiclassical limit in Quantum Mechanics, it has several shortcomings. First, the whole approach is technically difficult to grasp. Second, for several technical reasons, it leads to rather weak results when the potential function is analytic. Third, it seems to be unable to cope with resonant Schrödinger operators (as defined above). On the other hand, the study of the semiclassical limit of the time-dependent Schrödinger operator done by Hagedorn and Joye [8] seems to indicate that one should be able to deal with this problem in the much simpler framework of R-S perturbation theory, in particular when it comes to the analytic case. Also, the issues concerning the resonance condition should be, in principle, just eliminated by resorting to degenerated R-S perturbation theory.

This dissertation is organized as follows. In Chapter 2 we state the hypotheses of the problem, make a suitable transformation of equation (1.1), and prove some technical results. In Chapter 3 we construct some operators through recursion relations, which allow us to 
calculate the several correction terms involved in the formal series for eigenvalues and eigenvectors. In particular, this construction allows us to consider the cases where degeneracy occurs. Because of the transformation made in Chapter 2, we obtain a manageable recursion relation for the $n^{\text {th }}$ term of the R-S series. Then we state and prove an estimate to the growth of these terms. In Chapter 4 we define a residual error function for equation (1.1) and prove an estimate for it. The main results are stated and proved in Chapter 5. Finally, in Chapter 6 we summarize results and discuss possible generalizations. The Appendix is devoted to a computation needed in Chapter 4 . 


\section{Chapter 2}

\section{Preliminaries}

We shall assume that the potential energy $V(x)$ satisfies the following conditions:

H1 Let $V(x)$ be a $C^{\infty}$ real function on $\mathbb{R}^{d}$ such that $\liminf \operatorname{ix|\rightarrow \infty }_{\mid x} V(x)=: V_{\infty}>0$.

H2 $V(x)$ has a unique global minimum $V(0)=0$ at $x=0$.

H3 The global minimum of $V(x)$ is non-degenerate in the sense that

$$
\operatorname{Hess}_{V}(0)=\operatorname{diag}\left[\omega_{1}^{2}, \ldots, \omega_{d}^{2}\right]
$$

has only strictly positive eigenfrequencies $\omega_{1}, \ldots, \omega_{d}$. Let us denote the lowest eigenfrequency by $\omega_{0}$.

$\mathbf{H} 4 V(x)$ has an analytic extension to a neighborhood of the region $S_{\delta}=\left\{z:\left|\operatorname{Im} z_{i}\right| \leq \delta\right\}$ for some $\delta>0$. Without loss we may assume that $\delta<1$. 
H5 $V(z)$ satisfies $|V(z)| \leq M \exp \left(\tau|z|^{2}\right)$ uniformly in $S_{\delta}$, for some positive constants $M>0$ and $\omega_{0} / 4 \geq \tau>0$

According to Theorem XIII.15 of [23], hypotheses H1 implies that $\sigma_{\text {ess }}(H(\hbar))=\left[V_{\infty}, \infty\right)$. If moreover $V_{\infty}=\infty$, then $H(\hbar)$ has only purely discrete spectrum [23, Thm. XIII.16]. When $V_{\infty}<\infty$, one may only expect $H(\hbar)$ to have discrete eigenvalues inside $\left[0, V_{\infty}\right)$. As we have mentioned in the Introduction, existence of low-lying eigenvalues in the limit $\hbar \searrow 0$ has been proved, using different arguments, by Combes et al, Simon, and Howland. Among these, the formulation by Simon is the most general because his proof is valid for the multidimensional case. We reproduce the precise statement of this result below in Section 2.1.

The different proofs of existence of low-lying eigenvalues, inside a potential well, rely on the idea of splitting $H(\hbar)$ into a harmonic oscillator piece plus a residual which can be considered as a perturbation of it. For that reason the hypothesis H3 is critical. We also remark that the uniqueness of the global minimum in $\mathrm{H} 2$ is not necessary for those results to hold. Indeed, one of the main motivations to study the semiclassical limit of low-lying eigenvalues has been its connection with the problem of characterizing the semiclassical behavior of the discrete spectrum, when the phenomenon of tunneling plays a role. That is, when the potential energy has several global minima $[4,25,10]$. We include this uniqueness assumption in H2 in order to avoid the technical difficulties related to tunneling.

Hypotheses H4 and H5 are fundamental for the results to be discussed in this work. As we have mentioned in the Introduction, we want to develop a method to obtain exponen- 
tially accurate truncations of the Raleigh-Schrödinger series associated to the semiclassical eigensolutions. In order to obtain them, we rely heavily on the use of the Cauchy integral formula to control the behavior of the derivatives of $V(x)$. In Chapter 4 we shall estimate the error committed by inserting truncated series into the (rescaled) Schrödinger equation (2.1) defined below. This estimate involves the evaluation of integrals of the form

$$
\int_{\mathbb{R}^{d}} \operatorname{Polynomial}(x)\left|D^{\alpha} V(x)\right| e^{-c x^{2}} d^{d} x
$$

which crucially depends upon hypothesis H5. The question of whether or not one could use suitable cut-off functions to eliminate the need of this last assumption remains open.

Although the set of hypotheses $\mathrm{H} 1-\mathrm{H} 5$ seems to be quite restrictive, it leaves room for non-trivial realizations:

Example In $\mathbb{R}$, consider $V(x):=1-\left(1+x^{2}\right)^{-1} \cos (x)$. Clearly $V(x) \in C^{\infty}(\mathbb{R})$ with $V_{\infty}=1$. Also, $V(x)$ has a global minimum at the origin with $\omega^{2}:=V^{\prime \prime}(0)=3$. This function admits analytical extension into the open strip $\{z:|\operatorname{Im}(z)|<1\} \subset \mathbb{C}$.

Example $V(x, y):=1 / 2 \log \left(1+\omega_{1}^{2} x^{2}+\omega_{2}^{2} y^{2}\right)$. Then $V(x, y) \in C^{\infty}\left(\mathbb{R}^{2}\right)$ and $V_{\infty}=\infty$. The minimum of this function is located at the origin with $\operatorname{Hess}_{V}(0,0)=\operatorname{diag}\left[\omega_{1}^{2}, \omega_{2}^{2}\right]$. It can be extended to a holomorphic function in $\left\{\left(z_{1}, z_{2}\right) \in \mathbb{C}^{2}:\left|\operatorname{Im}\left(z_{1}\right)\right|<1\right.$ and $\left.\operatorname{Im}\left(z_{2}\right) \mid<1\right\}$.

Remark In this work we shall use the standard multi-index notation: for $\alpha=\left(\alpha_{1}, \ldots, \alpha_{d}\right) \in$ $\mathbb{N}^{d} \cup 0$ and $x=\left(x_{1}, \ldots, x_{d}\right) \in \mathbb{R}^{d}$, we denote $|\alpha|:=\alpha_{1}+\ldots+\alpha_{d}, \alpha !:=\alpha_{1} ! \cdot \ldots \cdot \alpha_{d} !$, $x^{\alpha}:=x_{1}^{\alpha_{1}} \cdot \ldots \cdot x_{d}^{\alpha_{d}}, D^{\alpha}:=\partial_{x_{1}}^{\alpha_{1}} \cdot \ldots \cdot \partial_{x_{d}}^{\alpha_{d}}$, and $x^{2}:=x_{1}^{2}+\ldots+x_{d}^{2}$. For $z=\left(z_{1}, \ldots, z_{d}\right) \in \mathbb{C}^{d}$, we denote $|z|^{2}:=z_{1} z_{1}^{*}+\ldots+z_{d} z_{d}^{*}$. 


\section{$2.1 \quad$ R-S perturbation theory}

We first transform (1.1) by scaling $x \rightarrow \hbar^{\frac{1}{2}} x$ and then dividing the whole equation by $\hbar$. This unitary transformation scales the eigenvalues and eigenfunctions as $E \rightarrow \hbar^{-1} E$ and $\tilde{\Psi}(x) \rightarrow \tilde{\Psi}(\sqrt{\hbar} x)$ respectively. The transformed equation may be written as

$$
\left[-\frac{1}{2} \Delta_{x}+V(\hbar ; x)\right] \tilde{\Psi}(\hbar ; x)=E(\hbar) \tilde{\Psi}(\hbar ; x)
$$

Because of hypothesis H3, $V(x)$ admits a Taylor expansion up to any order $n$. Thus we can write

$$
V(\hbar ; x)=\frac{1}{2} \sum_{i, j=1}^{d} A_{i j} x_{i} x_{j}+W(\hbar ; x)
$$

where the function $W(\hbar ; x)$ can be asymptotically approximated by

$$
W(\hbar ; x)=\sum_{l=3}^{n} \hbar^{\frac{l-2}{2}} \sum_{|\alpha|=l} \frac{D^{\alpha} V(0)}{\alpha !} x^{\alpha}+O\left(\hbar^{\frac{n-1}{2}} x^{|\alpha|=n+1}\right),
$$

Hypothesis H4 implies furthermore that the Taylor series (2.2) is convergent inside the open poly-disc $\left\{z \in \mathbb{C}^{d}:\left|z_{i}\right| \leq \delta\right\}$. Upper bounds on the derivatives of $V(x)$ can be easily obtained by using the Cauchy integral formula. They are stated and proved below in Lemma 2.2.

Now we can rewrite $(2.1)$ as

$$
\left[H_{0}+W(\hbar ; x)\right] \tilde{\Psi}(\hbar ; x)=E(\hbar) \tilde{\Psi}(\hbar ; x)
$$

where, in suitable cartesian coordinates,

$$
H_{0}=-\frac{1}{2} \Delta_{x}+\frac{1}{2} \sum_{i=1}^{d} \omega_{i}^{2} x_{i}^{2}
$$


is a harmonic oscillator hamiltonian with eigenfrequencies $\omega_{1}, \ldots, \omega_{d}$. The eigenfunctions of $H_{0}$ are therefore

$$
\Phi_{\alpha}(x)=\left(\pi^{-d} \prod_{i=1}^{d} \omega_{i}\right)^{\frac{1}{4}}\left(2^{|\alpha|} \alpha !\right)^{-\frac{1}{2}} \exp \left(-\frac{1}{2} \sum_{i=1}^{d} \omega_{i} x_{i}^{2}\right) \prod_{i=1}^{d} h_{\alpha_{i}}\left(\sqrt{\omega_{i}} x_{i}\right),
$$

where $h_{j}(y)$ denotes the Hermite polynomial of degree $j$. The corresponding eigenvalues are $e_{\alpha}=\sum_{i=1}^{d} \omega_{i} \alpha_{i}+d / 2$.

The fact that equation (2.1) admits solutions for small values $\hbar$ has been shown in several ways, as we have mentioned above. Here we reproduce the statement of this assertion as given by Simon in [24]:

Theorem 2.1 (Thm. 1.1 in [24]) Let $\left\{e_{I}\right\}_{I=0}^{\infty}$ be an increasing ordering of the eigenvalues of $H_{0}$, counting multiplicities. Assume $V(x)$ satisfies hypotheses H1-H3. Fix J. Then there exists $\hbar_{0}>0$ such that for each $0<\hbar \leq \hbar_{0}$ the equation (2.1) has at least $J$ solutions. Furthermore, the $J$ eigenvalues obey $\lim _{\hbar \rightarrow 0} E_{I}(\hbar)=e_{I}$.

In the semiclassical limit we want to consider $W(\hbar, x)$ as a perturbation of $H_{0}$. That raises the natural question of whether or not the low-lying eigenvalues and the corresponding eigenfunctions admit asymptotic series of the form

$$
\begin{aligned}
& \tilde{\Psi}(x) \sim \tilde{\psi}_{0}(x)+\hbar^{\frac{1}{2}} \tilde{\psi}_{1}(x)+\hbar^{\frac{2}{2}} \tilde{\psi}_{2}(x)+\hbar^{\frac{3}{2}} \tilde{\psi}_{3}(x)+\hbar^{\frac{4}{2}} \tilde{\psi}_{4}(x)+\ldots, \\
& E(\hbar) \sim \mathcal{E}_{0}+\hbar^{\frac{1}{2}} \mathcal{E}_{1}+\hbar^{\frac{2}{2}} \mathcal{E}_{2}+\hbar^{\frac{3}{2}} \mathcal{E}_{3}+\hbar^{\frac{4}{2}} \mathcal{E}_{4}+\ldots
\end{aligned}
$$

the so-called Rayleigh-Schrödinger series. The answer is yes, and is shown in $[3,24,11]$. For the multidimensional case, this statement is proved in [24], Theorem 5.1 and 5.3. 
In this work we essentially follow the standard, formal method to compute the R-S coefficients (see e. g., [17, Chapter XVI],) although alternatively we could use the technique developed by Kato [14, Chapters VII and VIII]. However, this last approach seems rather difficult to implement here, in particular when degeneracy occurs. Concerning asymptotics in degenerate perturbation theory, we must mention the approach developed by Hunziker-Pillet $[12,13]$.

In the first method mentioned above, one proposes formal R-S series, inserts them into (2.1) and equates powers of $\hbar^{\frac{1}{2}}$. The zeroth-order equation yields $H_{0} \psi_{0}=\mathcal{E}_{0} \psi_{0}$. Then $\mathcal{E}_{0}=e$ and $\psi_{0} \in G$, where $e$ is some eigenvalue of $H_{0}$ with multiplicity $g$ and associated eigenspace $G$. For $n=1,2, \ldots$, we have

$$
\left(H_{0}-e\right) \tilde{\psi}_{n}+\sum_{l=1}^{n} \tilde{T}^{(l+2)} \tilde{\psi}_{n-l}=\sum_{l=1}^{n} \mathcal{E}_{l} \tilde{\psi}_{n-l}
$$

where we define

$$
\tilde{T}^{(l)}:=\sum_{|\alpha|=l} \frac{1}{\alpha !} D^{\alpha} V(0) x^{\alpha} .
$$

A simple yet important property of the correction terms $\tilde{\psi}_{n}$ is the following:

Lemma 2.1 Let $P_{|\alpha| \leq l}$ be the projection onto the subspace spanned by $\left\{\Phi_{\alpha}:|\alpha| \leq l\right\}$ and $a=a_{e}$ be the smallest non-negative integer such that $G \subseteq \operatorname{Ran}\left(P_{|\alpha| \leq a}\right)$. Then, for each $n \geq 1, \tilde{\psi}_{n} \in \operatorname{Ran}\left(P_{|\alpha| \leq a+3 n}\right)$

Proof. First, decompose $\tilde{\psi}_{n}=P_{|\alpha| \leq a} \tilde{\psi}_{n}+\left(1-P_{|\alpha| \leq a}\right) \tilde{\psi}_{n}=: \tilde{\psi}_{n}^{(1)}+\tilde{\psi}_{n}^{(2)}$. We have to prove 
the assertion only for $\tilde{\psi}_{n}^{(2)}$. Equation (2.7) yields

$$
\tilde{\psi}_{n}^{(2)}=\left(H_{0}-e\right)_{r}^{-1}\left(1-P_{|\alpha| \leq a}\right)\left[\sum_{l=1}^{n} \mathcal{E}_{l} \tilde{\psi}_{n-l}-\sum_{l=1}^{n} \tilde{T}^{(l+2)} \tilde{\psi}_{n-l}\right]
$$

where $\left(H_{0}-e\right)_{r}^{-1}$ is the inverse of the restriction of $H_{0}-e$ onto Ran $\left(1-P_{|\alpha| \leq a}\right)$. Since

$$
\operatorname{Ran}\left(\left(H_{0}-e\right)_{r}^{-1}\left(1-P_{|\alpha| \leq a}\right) P_{|\alpha| \leq a+3 n}\right) \subset \operatorname{Ran}\left(P_{|\alpha| \leq a+3 n}\right)
$$

it is sufficient to show that

$$
\left(\sum_{l=1}^{n} \mathcal{E}_{l} \tilde{\psi}_{n-l}-\sum_{l=1}^{n} \tilde{T}^{(l+2)} \tilde{\psi}_{n-l}\right) \in P_{|\alpha| \leq a+3 n} .
$$

Now use mathematical induction. For $n=1$, the assertion $\tilde{T}^{(3)} \tilde{\psi}_{0} \in P_{|\alpha| \leq a+3}$ follows from the fact that $\tilde{T}^{(3)}$ contains terms that are at most proportional to the third power of creation operators, and that $\tilde{\psi}_{0} \in G \subset P_{|\alpha| \leq a}$. Assuming that statement is true for $s=1, \ldots, n-1$, then it is trivially true for the first term in (2.8). Also, a simple calculation with ladder operators shows that $x^{\alpha} \varphi \in \operatorname{Ran}\left(P_{|\beta| \leq a+3(n-l)+|\alpha|}\right)$ whenever $\varphi \in \operatorname{Ran}\left(P_{|\beta| \leq a+3(n-l)}\right)$. Finally, we have $3(n-l)+2+l=3 n+2(1-l) \leq 3 n$ for $l=1, \ldots, n$.

The set of recursive equations (2.7) is not suitable for the purpose of finding the sharp upper bounds for the R-S coefficients that we shall need later. It turns out to be convenient to transform the problem in the following way: Let $\left\{\Phi_{\alpha}(x)\right\}$ be a basis of eigenvectors of $H_{0}$. For a given eigenvalue $e$ of $H_{0}$, let us define a new operator $A_{e}$ by

$$
A_{e} \Phi_{\alpha}(x)= \begin{cases}\Phi_{\alpha}(x) & \text { if } \Phi_{\alpha}(x) \in G \\ \left|e-e_{\alpha}\right|^{-\frac{1}{2}} \Phi_{\alpha}(x) & \text { otherwise, }\end{cases}
$$


where $e_{\alpha}$ is the eigenvalue associated to $\Phi_{\alpha}(x)$. Then extend $A_{e}$ to the whole Hilbert space $\mathcal{H}$ by linearity. So defined, $A_{e}$ is a bounded operator with unit norm but unbounded inverse. However, $\operatorname{Ran}\left(P_{|\alpha| \leq a+3 n}\right)$ is clearly in the domain of $A_{e}^{-1}$ for each $n \in \mathbb{N}$. This fact allows us to consider the equivalent set of equations

$$
H_{e} \psi_{n}+\sum_{l=1}^{n} T^{(l+2)} \psi_{n-l}=\sum_{l=1}^{n} \mathcal{E}_{l} A_{e}^{2} \psi_{n-l}
$$

where $H_{e}:=A_{e}\left(H_{0}-e\right) A_{e}, T^{(m)}:=A_{e} \tilde{T}^{(m)} A_{e}$, and $\psi_{m}=A_{e}^{-1} \tilde{\psi}_{m}$. The operator $H_{e}$ satisfies

$$
H_{e} \Phi_{\alpha}(x)= \begin{cases}0 & \text { if } \Phi_{\alpha}(x) \in G \\ \frac{e-e_{\alpha}}{\left|e-e_{\alpha}\right|} \Phi_{\alpha}(x) & \text { otherwise. }\end{cases}
$$

Therefore the norm of $H_{e}$ is equal to 1 . In Chapter 3 we shall prove that both $\left|\mathcal{E}_{n}\right|$ and $\left\|\psi_{n}\right\|$ essentially grow as $b^{n} \sqrt{n !}$ for large $n$.

\subsection{Some technical results}

We conclude this chapter with an assortment of technical lemmas. Lemma 2.2 states certain estimates on the derivatives of the potential energy. In Lemma 2.3 we show a key upper bound to the norm of the operators $T^{(l)} P_{|\alpha| \leq n}$. Finally, in Lemma 2.5 we state results about certain expressions involving factorials that we shall use extensively in the sequel.

Lemma 2.2 Assume $V(x)$ satisfies $H_{4}$. Then there are constants $C_{1}$ and $C_{2}$ such that, for $l \geq 1$

$$
\sum_{|\alpha|=l} \frac{\left|D^{\alpha} V(0)\right|}{\alpha !} \delta^{|\alpha|} \leq C_{1} C_{2}^{l}
$$


If $V(x)$ also satisfies $H 5$ then there exists a constant $C_{0}$ such that

$$
\frac{\delta^{|\alpha|}}{\alpha !}\left|D^{\alpha} V(x)\right| \leq C_{0} \exp \left(2 \tau x^{2}\right)
$$

Proof. Let $\Gamma_{i}$ be a circle of radius $\delta$ in the complex plane, centered at $x_{i}$. Then the Cauchy integral formula applied to $V(x)$, which makes sense because of hypothesis H4, states that for each multi-index $\alpha=\left(\alpha_{1}, \ldots, \alpha_{d}\right)$

$$
D^{\alpha} V(x)=\frac{\alpha !}{(2 \pi i)^{d}} \int_{\Gamma_{1}} d z_{1} \ldots \int_{\Gamma_{d}} d z_{d} \frac{V(z)}{\prod_{i=1}^{d}\left(z_{i}-x_{i}\right)^{\alpha_{i}+1}}
$$

which implies

$$
\left|D^{\alpha} V(x)\right| \leq \frac{\alpha !}{\delta^{|\alpha|}} \max _{z_{i} \in \Gamma_{i}}|V(z)|
$$

Let us prove (2.10) first. Because of H5,

$$
\max _{z_{i} \in \Gamma_{i}}|V(z)| \leq M \prod_{i=1}^{d} \max _{z_{i} \in \Gamma_{i}} \exp \left(\tau\left|z_{i}\right|^{2}\right) \leq M \prod_{i=1}^{d} \exp \left(\tau\left|x_{i}+\delta\right|^{2}\right) \leq M \exp \left(2 d \tau \delta^{2}\right) \exp \left(2 \tau x^{2}\right)
$$

so (2.11) implies (2.10), after defining $C_{0}=M \exp \left(2 d \tau \delta^{2}\right)$. If now the $\Gamma_{i}$ 's are circles centered at zero, we have (without assuming H5)

$$
\frac{\left|D^{\alpha} V(0)\right|}{\alpha !} \delta^{|\alpha|} \leq \max _{z_{i} \in \Gamma_{i}}|V(z)|=: c<\infty .
$$

Then

$$
\sum_{|\alpha|=l} \frac{\left|D^{\alpha} V(0)\right|}{\alpha !} \delta^{|\alpha|} \leq c \sum_{|\alpha|=l} 1
$$

for all $l$. The last summation is the number of different ways to sum $d$ non-negative integers such as the result is equal to $l$. That is,

$$
\sum_{|\alpha|=l} 1=\frac{(l+d-1) !}{l !(d-1) !} \leq \frac{1}{(d-1) !}(l+d-1)^{d-1}
$$


Therefore, we have

$$
\sum_{|\alpha|=l} \frac{\left|D^{\alpha} V(0)\right|}{\alpha !} \delta^{|\alpha|} \leq \frac{c}{(d-1) !}(l+d-1)^{d-1} \leq C_{1} C_{2}^{l}
$$

with obvious definition of $C_{1}$, and $C_{2}$ being either equal to $(d-1) \max _{l \geq 1} \log (l+d-1) / l$ (when $d>1$ ) or equal to 1 (when $d=1$ ).

Lemma 2.3 For $|\alpha| \geq 2, n \geq 0$ and some constant $\gamma>0$,

$$
\left\|A_{e} x^{\alpha} A_{e} P_{|\alpha| \leq n}\right\| \leq \gamma^{2}\left(\frac{2}{\omega_{0}}\right)^{\frac{|\alpha|-2}{2}}\left[\frac{(n+|\alpha|-1) !}{(n+1) !}\right]^{\frac{1}{2}} .
$$

As a consequence,

$$
\left\|T^{(l)} P_{|\alpha| \leq n}\right\| \leq C_{3} \kappa^{\frac{l-2}{2}}\left[\frac{(n+l-1) !}{(n+1) !}\right]^{\frac{1}{2}}
$$

for some $C_{3}>0$ and $\kappa \geq 2$.

Recall that $\omega_{0}$ is the lowest eigenfrequency of $H_{0}$. To prove the first inequality of Lemma 2.3, we resort to a slightly modified version of a result by Hagedorn and Joye [8]. For a sake of completeness, we state it here:

Lemma 2.4 (Lemma 5.1 in [8]) In d dimensions,

$$
x^{\alpha} P_{|\beta| \leq m}=P_{|\beta| \leq m+|\alpha|} x^{\alpha} P_{|\beta| \leq m}
$$

and

$$
\left\|x^{\alpha} P_{|\beta| \leq m}\right\| \leq\left(\frac{2}{\omega_{0}}\right)^{\frac{|\alpha|}{2}}\left[\frac{(m+|\alpha|) !}{m !}\right]^{\frac{1}{2}} .
$$


Proof. For a single coordinate $x_{i}$, we have

$$
x_{i}=\frac{1}{\sqrt{2 \omega_{i}}}\left(a_{i}+a_{i}^{*}\right)
$$

where $a_{i}$ and $a_{i}^{*}$ are the associated ladder operators. It is straightforward to see that $a_{i} P_{|\beta| \leq k} \subset \operatorname{Ran}\left(P_{|\beta| \leq k-1}\right), a_{i}^{*} P_{|\beta| \leq k} \subset \operatorname{Ran}\left(P_{|\beta| \leq k+1}\right)$, and then $x_{i} P_{|\beta| \leq k} \subset \operatorname{Ran}\left(P_{|\beta| \leq k+1}\right)$. Now consider any vector $\varphi \in \operatorname{Ran}\left(P_{|\beta| \leq k}\right)$. It follows that $\left\|a_{i} P_{|\beta| \leq k} \varphi\right\| \leq \sqrt{k}\|\varphi\|$ and also $\left\|a_{i}^{*} P_{|\beta| \leq k} \varphi\right\| \leq \sqrt{k+1}\|\varphi\|$, which imply that $\left\|x_{i} P_{|\beta| \leq k}\right\| \leq \sqrt{2(k+1) / \omega_{0}}$. Now use induction.

Proof of Lemma 2.3. We start again from (2.12). Consider any $\varphi=\sum_{\beta} d_{\beta} \Phi_{\beta} \in \mathcal{H}$.

Define $J_{G}:=\left\{\right.$ multi-indices $\left.\beta: \Phi_{\beta} \in G\right\}$. Then

$$
\begin{aligned}
a_{i}^{*} A_{e} \varphi & =\sum_{\beta \in J_{G}} d_{\beta} a_{i}^{*} \Phi_{\beta}+\sum_{\beta \notin J_{G}} d_{\beta}\left|e-e_{\beta}\right|^{-\frac{1}{2}} a_{i}^{*} \Phi_{\beta} \\
& =\sum_{\beta \in J_{G}} d_{\beta} \sqrt{\beta_{i}+1} \Phi_{\beta+1_{i}}+\sum_{\beta \notin J_{G}} d_{\beta}\left|e-e_{\beta}\right|^{-\frac{1}{2}} \sqrt{\beta_{i}+1} \Phi_{\beta+1_{i}}
\end{aligned}
$$

where $\beta+1_{i}:=\left(\beta_{1}, \ldots, \beta_{i}+1, \ldots, \beta_{d}\right)$. Thus,

$$
\begin{aligned}
\left\|a_{i}^{*} A_{e} \varphi\right\|^{2} & =\sum_{\beta \in J_{G}}\left|d_{\beta}\right|^{2}\left(\beta_{i}+1\right)+\sum_{\beta \notin J_{G}}\left|d_{\beta}\right|^{2}\left|e-e_{\beta}\right|^{-1}\left(\beta_{i}+1\right) \\
& \leq(1+a) \sum_{\beta \in J_{G}}\left|d_{\beta}\right|^{2}+\sum_{\beta \notin J_{G}}\left|d_{\beta}\right|^{2}\left|e-e_{\beta}\right|^{-1}\left(\beta_{i}+1\right)
\end{aligned}
$$

because $\beta \in J_{G}$ implies $\beta_{i} \leq|\beta| \leq a$. Moreover,

$$
\frac{\beta_{i}+1}{\left|e-e_{\beta}\right|}=\frac{1}{\omega_{i}} \frac{\omega_{i}\left(\beta_{i}+1 / 2\right)}{\left|e-e_{\beta}\right|}+\frac{1 / 2}{\left|e-e_{\beta}\right|} \leq \frac{1}{\omega_{i}} \frac{e_{\beta}}{\left|e-e_{\beta}\right|}+\frac{1 / 2}{\left|e-e_{\beta}\right|}
$$

Since $\sigma\left(H_{0}\right)$ has no accumulation points and $e_{\beta} \neq e$ for all $\beta \notin J_{G}$, $\inf _{\beta \notin J_{G}}\left|e-e_{\beta}\right|>0$.

Furthermore, since $\lim _{|\beta| \rightarrow \infty} e_{\beta}\left|e-e_{\beta}\right|^{-1}=1, \sup _{\beta \notin J_{G}} e_{\beta}\left|e-e_{\beta}\right|^{-1}<\infty$. Thus,

$$
\left|e-e_{\beta}\right|^{-1}\left(\beta_{i}+1\right) \leq \frac{1}{\omega_{i}} \sup _{\beta \notin J_{G}} e_{\beta}\left|e-e_{\beta}\right|^{-1}+\frac{1}{2} \sup _{\beta \notin J_{G}}\left|e-e_{\beta}\right|^{-1}=: K_{1}<\infty
$$


which implies

$$
\left\|a_{i}^{*} A_{e}\right\|^{2} \leq \max \left\{(1+a), K_{1}\right\} \leq \max _{\left\{\omega_{i}\right\}} \max \left\{(1+a), K_{1}\right\}
$$

A similar calculation yields,

$$
\left\|a_{i} A_{e}\right\|^{2} \leq \max \left\{|1-a|, K_{2}\right\} \leq \max _{\left\{\omega_{i}\right\}} \max \left\{|1-a|, K_{2}\right\}
$$

for some $K_{2}<\infty$. Therefore,

$$
\left\|x_{i} A_{e}\right\| \leq \frac{1}{\sqrt{2 \omega_{i}}}\left\|a_{i} A_{e}\right\|+\frac{1}{\sqrt{2 \omega_{i}}}\left\|a_{i}^{*} A_{e}\right\| \leq \frac{1}{\sqrt{2 \omega_{0}}}\left(\left\|a_{i} A_{e}\right\|+\left\|a_{i}^{*} A_{e}\right\|\right) \leq \gamma
$$

where $\omega_{0}$ is the lowest eigenfrequency of $H_{0}$, and we use the sum of the right-hand sides of (2.13) and (2.14) to define $\gamma$. Taking the adjoint yields

$$
\left\|A_{e} x_{i}\right\| \leq \gamma
$$

Since $|\alpha| \geq 2$, we can write $x^{\alpha}=x_{i} x^{\alpha^{\prime}} x_{j}$ for some $x_{i}, x_{j}$, with $\left|\alpha^{\prime}\right|=|\alpha|-2$. Then

$$
\begin{aligned}
\left\|A_{e} x^{\alpha} A_{e} P_{|\beta| \leq n}\right\| & \leq\left\|A_{e} x_{i} x^{\alpha^{\prime}} P_{|\beta| \leq n+1} x_{j} A_{e} P_{|\beta| \leq n}\right\| \\
& \leq\left\|A_{e} x_{i}\right\|\left\|x_{j} A_{e}\right\|\left\|x^{\alpha^{\prime}} P_{|\beta| \leq n+1}\right\| \\
& \leq \gamma^{2}\left(\frac{2}{\omega_{0}}\right)^{\frac{\left|\alpha^{\prime}\right|}{2}}\left[\frac{\left(n+\left|\alpha^{\prime}\right|+1\right) !}{(n+1) !}\right]^{\frac{1}{2}} \\
& =\gamma^{2}\left(\frac{2}{\omega_{0}}\right)^{\frac{|\alpha|-2}{2}}\left[\frac{(n+|\alpha|-1) !}{(n+1) !}\right]^{\frac{1}{2}}
\end{aligned}
$$

where we use Lemma 5.1 of $[8]$ to bound $\left\|x^{\alpha^{\prime}} P_{|\beta| \leq n+1}\right\|$. The last statement follows from the definition of $T^{(l)}$ and the first part of Lemma 2.2, along with the definitions $C_{3}=C_{1} \gamma^{2} \delta^{-2} C_{2}^{2}$ and $\kappa=\max \left\{2,2 \omega_{0}^{-1} \delta^{-2} C_{2}^{2}\right\}$.

Lemma 2.5 Let $\kappa \geq 2$ be the number defined in Lemma 2.3. Then 
1. For each integer $a \geq 0$ there is a constant $C_{4}=C_{4}(a)$ so that, for all $m \geq 0$,

$$
\sum_{l=0}^{m}\left[\frac{(1+a+m-l) !(1+a+l) !}{(1+a+m) !}\right]^{\frac{1}{2}} \leq C_{4}
$$

2. For all $a \geq-1$ there is a constant $C_{5}$ so that, for all $m \geq 0$,

$$
\sum_{l=0}^{m} \kappa^{-\frac{5 l}{2}}\left[\frac{(1+a+3 m-2 l) !(1+a+m-l) !}{(1+a+3 m-3 l) !(1+a+m) !}\right]^{\frac{1}{2}} \leq C_{5}
$$

3. For each $a \geq 0$ there is a constant $C_{6}=C_{6}(a)$ so that, for all $m \geq 0$,

$$
\sum_{l=1}^{m} \kappa^{-\frac{5 l}{2}}\left[\frac{(1+a+m-l) !(1+a+l) !}{(1+a) !(a+m) !}\right]^{\frac{1}{2}} \leq C_{6}
$$

Proof. (1) The statement is obviously true for $n=0,1,2,3$. For $n \geq 4$,

$$
\begin{aligned}
\sum_{l=0}^{m}\left[\frac{(1+a+m-l) !(1+a+l) !}{(1+a+m) !}\right]^{\frac{1}{2}} & \\
= & 2[(1+a) !]^{\frac{1}{2}}+2\left[\frac{(2+a) !}{1+a+m}\right]^{\frac{1}{2}}+\sum_{l=2}^{m-2}\left[\frac{(1+a+m-l) !(1+a+l) !}{(1+a+m) !}\right]^{\frac{1}{2}} \\
\leq & 2[(1+a) !]^{\frac{1}{2}}+2\left[\frac{(2+a) !}{1+a}\right]^{\frac{1}{2}} \\
& +(m-3) \max _{2 \leq l \leq \llbracket \frac{m}{2} \rrbracket}\left[\frac{(1+a+m-l) !(1+a+l) !}{(1+a+m) !}\right]^{\frac{1}{2}}
\end{aligned}
$$

where $\llbracket J \rrbracket$ denotes the greatest integer less than or equal to $J$. Since $(1+a+m-l) !(1+a+l)$ ! is decreasing for $l \leq \llbracket \frac{m}{2} \rrbracket$, it follows that

$$
\sum_{l=0}^{m}\left[\frac{(1+a+m-l) !(1+a+l) !}{(1+a+m) !}\right]^{\frac{1}{2}} \leq 2[(1+a) !]^{\frac{1}{2}}+2\left[\frac{(2+a) !}{1+a}\right]^{\frac{1}{2}}+[(3+a) !]^{\frac{1}{2}} \frac{m-3}{m+a} .
$$

The last term converges as $m \rightarrow \infty$, so existence of the constant $C_{4}(a)$ is guaranteed. 
(2) By cancelling common factors, we have

$$
\sum_{l=0}^{m} \kappa^{-\frac{5 l}{2}} \prod_{s=1}^{l}\left(\frac{1+a+3 m-3 l+s}{1+a+m-l+s}\right)^{\frac{1}{2}}
$$

For $a \geq-1$ and $s \geq 0$, we have $0 \leq 2(1+a+s)$. This implies

$$
\frac{1+a+3 m-3 l+s}{1+a+m-l+s} \leq 3 .
$$

Therefore,

$$
\sum_{l=0}^{m} \kappa^{-\frac{5 l}{2}}\left[\frac{(1+a+3 m-2 l) !(1+a+m-l) !}{(1+a+3 m-3 l) !(1+a+m) !}\right]^{\frac{1}{2}} \leq \sum_{l=0}^{m} \kappa^{-\frac{5 l}{2}} 3^{\frac{l}{2}}
$$

and the right hand side converges to $C_{5}=\left(1-\sqrt{3 / \kappa^{5}}\right)^{-1}$.

(3) Notice that for $1 \leq l \leq m-1$ we have

$$
\frac{(1+a+l) !(1+a+m-l) !}{(a+m) !(1+a) !}=(1+a+l) \frac{\prod_{s=1}^{m-l}(1+a+s)}{\prod_{s=l}^{m-1}(1+a+s)}=(1+a+l) \prod_{s=1}^{m-l} \frac{1+a+s}{l+a+s} \leq 1+a+l .
$$

Therefore

$$
\sum_{l=1}^{m} \kappa^{-\frac{5 l}{2}}\left[\frac{(1+a+l) !(1+a+m-l) !}{(a+m) !(1+a) !}\right]^{\frac{1}{2}} \leq \sum_{l=1}^{m} \kappa^{-\frac{5 l}{2}}(1+a+l)^{\frac{1}{2}}
$$

where the right-hand side converges to some constant $C_{6}(a)<\infty$. 


\section{Chapter 3}

\section{Computation of the R-S coefficients}

Let us assume that the zeroth-order eigenvalue $e$ is $g$-fold degenerate, with associated eigenspace $G$. We allow $g$ to be equal to 1 . Let $\mathrm{P}$ be the projector onto $G$ and $Q:=1-P$. Up to zeroth-order, $\psi_{0}$ can be any vector in $G$, which we may require to be normalized, $\left\|\psi_{0}\right\|=1$. Two cases may arise from solving (2.9) at higher order. Either the zeroth-order degeneracy is preserved at all orders, or it is removed to some extent at higher order. Let us start by discussing the former case, which trivially includes the non-degenerate one.

\subsection{Degeneracy is preserved.}

Fix $\psi_{0} \in G$, with $\left\|\psi_{0}\right\|=1$. The first-order equation is

$$
H_{e} \psi_{1}+T^{(3)} \psi_{0}=\mathcal{E}_{1} A_{e}^{2} \psi_{0}
$$


Let us multiply by $P$. Noting that $P H_{e}=0$ and $P A_{e}^{2} \psi_{0}=\psi_{0}$, we obtain

$$
P T^{(3)} P \psi_{0}=\mathcal{E}_{1} \psi_{0}
$$

This is the secular equation for the finite-dimensional, self-adjoint operator $\Lambda^{(1)}:=P T^{(3)} P$. Since we assume that the zeroth-order degeneracy is not broken at any order, $\Lambda^{(1)}$ must have only one eigenvalue. Let us call it $\lambda_{1}$. Then $\mathcal{E}_{1}=\lambda_{1}$. Now multiply (3.1) by $Q$. We obtain

$$
H_{e} Q \psi_{1}=-Q T^{(3)} \psi_{0}
$$

Let us introduce more notation. For any vector $\psi \in \mathcal{H}$, define $\psi^{\|}:=P \psi$ and $\psi^{\perp}:=Q \psi$. Also, let $\left(H_{e}\right)_{\perp}$ be the restriction of $H_{e}$ to $\operatorname{Ran}(Q)$. So defined, $\left(H_{e}\right)_{\perp}$ is invertible. Then we have

$$
\psi_{1}^{\perp}=\Xi^{(1, \perp)} \psi_{0}
$$

where $\Xi^{(1, \perp)}:=\left(H_{e}\right)_{\perp}^{-1}\left(-Q T^{(3)}\right)$. So far $\psi_{1}^{\|}$remains undefined.

The second-order equation is

$$
H_{e} \psi_{2}+T^{(3)} \psi_{1}+T^{(4)} \psi_{0}=\mathcal{E}_{2} A_{e}^{2} \psi_{0}+\lambda_{1} A_{e}^{2} \psi_{1}
$$

Multiply (3.2) by $P$. After some algebra involving the definitions of $\Lambda^{(1)}$ and $\Xi^{(1, \perp)}$, we obtain

$$
\left(P T^{(3)} \Xi^{(1, \perp)} P+P T^{(4)} P\right) \psi_{0}=\mathcal{E}_{2} \psi_{0}
$$

Then $\mathcal{E}_{2}$ has to be equal to the unique eigenvalue of

$$
\Lambda^{(2)}:=P\left(T^{(3)} \Xi^{(1, \perp)}+T^{(4)}\right) P .
$$


That is, $\mathcal{E}_{2}=\lambda_{2}$. Now multiply (3.2) by $Q$ to obtain

$$
H_{e} \psi_{2}^{\perp}+Q T^{(3)}\left(\psi_{1}^{\|}+\psi_{1}^{\perp}\right)+Q T^{(4)} \psi_{0}=\lambda_{1} A_{e}^{2} \psi_{1}^{\perp}
$$

which yields

$$
\psi_{2}^{\perp}=\Xi^{(2, \perp)} \psi_{0}+\Xi^{(1, \perp)} \psi_{1}^{\|}
$$

where we define

$$
\Xi^{(2, \perp)}:=\left(H_{e}\right)_{\perp}^{-1}\left[\left(\lambda_{1} A_{e}^{2}-Q T^{(3)}\right) \Xi^{(1, \perp)}+Q T^{(4)}\right]
$$

and no requirement is imposed on either $\psi_{2}^{\|}$or $\psi_{1}^{\|}$.

The third-order equation is

$$
H_{e} \psi_{3}+T^{(3)} \psi_{2}+T^{(4)} \psi_{1}+T^{(5)} \psi_{0}=\mathcal{E}_{3} A_{e}^{2} \psi_{0}+\lambda_{2} A_{e}^{2} \psi_{1}+\lambda_{1} A_{e}^{2} \psi_{2}
$$

Following the procedure already described, we obtain

$$
\Lambda^{(3)} \psi_{0}=\mathcal{E}_{3} \psi_{0}
$$

where

$$
\Lambda^{(3)}:=P\left(T^{(3)} \Xi^{(2 \perp)}+T^{(4)} \Xi^{(1, \perp)}+T^{(5)}\right) P
$$

has only one eigenvalue $\lambda_{3}$. Thus $\mathcal{E}_{3}=\lambda_{3}$. Also

$$
\psi_{3}^{\perp}=\Xi^{(3, \perp)} \psi_{0}+\Xi^{(2, \perp)} \psi_{1}^{\|}+\Xi^{(1, \perp)} \psi_{2}^{\|}
$$

where

$$
\Xi^{(3, \perp)}:=\left(H_{e}\right)_{\perp}^{-1}\left[\left(\lambda_{1} A_{e}^{2}-Q T^{(3)}\right) \Xi^{(2, \perp)}+\left(\lambda_{2} A_{e}^{2}-Q T^{(4)}\right) \Xi^{(1, \perp)}-Q T^{(5)}\right]
$$


and nothing is said about $\psi_{3}^{\|}, \psi_{2}^{\|}$or $\psi_{1}^{\|}$.

As one can see, $\mathcal{E}_{n}$ and $\psi_{n}^{\perp}$ can be calculated through recursive definition of certain operators.

The form of these operators is now easy to guess:

Proposition 3.1 For $n=1,2, \ldots$, recursively define

$$
\begin{aligned}
& \Xi^{(1, \perp)}:=-\left(H_{e}\right)_{\perp}^{-1} Q T^{(3)} \\
& \Xi^{(n, \perp)}:=\left(H_{e}\right)_{\perp}^{-1}\left[-Q T^{(n+2)}+\sum_{p=1}^{n-1}\left(\lambda_{n-p} A_{e}^{2}-Q T^{(n+2-p)}\right) \Xi^{(p, \perp)}\right]
\end{aligned}
$$

where $\lambda_{l}$ is, by assumption, the unique eigenvalue of

$$
\Lambda^{(l)}:=P T^{(l+2)} P+\sum_{p=1}^{n-1} P T^{(l+2-p)} \Xi^{(p, \perp)} P .
$$

Then, given $\psi_{0} \in G, \mathcal{E}_{n}=\lambda_{n}$ and

$$
\psi_{n}=\Xi^{(n, \perp)} \psi_{0}+\sum_{p=1}^{n-1} \Xi^{(n-p, \perp)} \psi_{p}^{\|}+\psi_{n}^{\|}
$$

where $\psi_{1}^{\|}, \ldots, \psi_{n}^{\|}$are vectors arbitrarily chosen from $G$.

This construction will be generalized in Proposition 3.2, from which the proof of Proposition 3.1 can be easily read out. To rule out arbitrariness, we set $\psi_{n}^{\|}=0$ for all $n \geq 1$, which is equivalent to absorbing those vectors into $\psi_{0}$ and renormalizing.

The recursive expressions for the operators $\Lambda^{(n)}$ and $\Xi^{(n, \perp)}$ can be translated into recursive expressions for $\mathcal{E}_{n}$ and $\psi_{n}$. The result is

$$
\begin{aligned}
& \mathcal{E}_{n}=\sum_{p=0}^{n-1}\left\langle T^{(n+2-p)} P_{|\alpha| \leq a} \psi_{0}, \psi_{p}\right\rangle \\
& \psi_{n}=\left(H_{e}\right)_{\perp}^{-1}\left[-Q T^{(n+2)} \psi_{0}+\sum_{p=1}^{n-1}\left(\mathcal{E}_{n-p} A_{e}^{2}-Q T^{(n+2-p)}\right) \psi_{p}\right] .
\end{aligned}
$$


Furthermore, we can easily obtain the following inequalities:

$$
\begin{aligned}
\left|\mathcal{E}_{n}\right| & \leq \sum_{l=1}^{n}\left\|T^{(l+2)} P_{|j| \leq a}\right\|\left\|\psi_{n-l}\right\| \\
\left\|\psi_{n}\right\| & \leq \sum_{l=1}^{n-1}\left|\mathcal{E}_{l}\right|\left\|\psi_{n-l}\right\|+\sum_{l=1}^{n}\left\|T^{(l+2)} P_{|j| \leq a+3(n-l)}\right\|\left\|\psi_{n-l}\right\| .
\end{aligned}
$$

By resorting to Lemma 2.3, we finally obtain

$$
\begin{aligned}
\left|\mathcal{E}_{n}\right| & \leq C_{3} \sum_{l=1}^{n} \kappa^{\frac{l}{2}}\left[\frac{(1+a+l) !}{(1+a) !}\right]^{\frac{1}{2}}\left\|\psi_{n-l}\right\| \\
\left\|\psi_{n}\right\| & \leq \sum_{l=1}^{n-1}\left|\mathcal{E}_{l}\right|\left\|\psi_{n-l}\right\|+C_{3} \sum_{l=1}^{n} \kappa^{\frac{l}{2}}\left[\frac{(1+a+3 n-2 l) !}{(1+a+3 n-3 l) !}\right]^{\frac{1}{2}}\left\|\psi_{n-l}\right\| .
\end{aligned}
$$

As an immediate consequence, we have

Theorem 3.1 For each $a \geq 0$, there is $b>0$ so that

$$
\begin{aligned}
\left|\mathcal{E}_{n}\right| & \leq \kappa^{3 n} b^{n}[(1+a+n) !]^{\frac{1}{2}} \\
\left\|\psi_{n}\right\| & \leq \kappa^{3 n} b^{n}[(1+a+n) !]^{\frac{1}{2}}
\end{aligned}
$$

for all $n \geq 1$.

A proof of this theorem is in [27], where the somewhat simpler one-dimensional problem is discussed. Alternatively, one can modify the proof of Theorem 3.2 below to get somewhat tighter bounds. 


\subsection{Degeneracy is removed.}

Let us examine the case where the zeroth-order degeneracy is partially removed only at first order.

First-order: Now the operator $\Lambda^{(1)}=P T^{(3)} P$ has $k \geq 2$ distinct eigenvalues $\lambda_{1,1}, \ldots, \lambda_{1, k}$. Let $G_{1}, \ldots, G_{k}$ be the corresponding eigenspaces, and let $P^{(1)}, \ldots, P^{(k)}$ be their orthogonal projections. Set $\mathcal{E}_{1}=\lambda_{1, i}$. Then $\psi_{0}$ must lie in $G_{i}$. As before, $\psi_{1}^{\perp}=\Xi^{(1, \perp)} \psi_{0}$ with $\Xi^{(1, \perp)}:=$ $\left(H_{e}\right)_{\perp}^{-1}\left(-Q T^{(3)}\right)$.

Second-order: Because of the choice for $\mathcal{E}_{1}$ we have

$$
H_{e} \psi_{2}+T^{(3)} \psi_{1}+T^{(4)} \psi_{0}=\mathcal{E}_{2} A_{e}^{2} \psi_{0}+\lambda_{1, i} A_{e}^{2} \psi_{1}
$$

Multiply (3.3) by $P^{(j)}$

$$
P^{(j)} T^{(3)} \psi_{1}+P^{(j)} T^{(4)} \psi_{0}=\mathcal{E}_{2} P^{(j)} \psi_{0}+\lambda_{1, i} P^{(j)} \psi_{1}
$$

Note that $P=\sum_{j=1}^{k} P^{(j)}$. Then, for any vector $\psi$, we have $\psi^{\|}=\sum_{j=1}^{k} \psi^{(j)}$. On the other hand,

$$
\begin{aligned}
P^{(j)} T^{(3)} \psi^{\|} & =\sum_{l=1}^{k} P^{(j)} P T^{(3)} P P^{(l)} \psi^{\|} \\
& =\sum_{l=1}^{k} P^{(j)} \Lambda^{(1)} P^{(l)} \psi^{\|} \\
& =\sum_{l=1}^{k} \lambda_{1, l} P^{(j)} P^{(l)} \psi^{\|} \\
& =\lambda_{1, j} \psi^{(j)} .
\end{aligned}
$$


Therefore, $\sum_{l \neq i} P^{(i)} T^{(3)} \psi_{n}^{(l)}=0$. The identity (3.5) yields

$$
\begin{aligned}
P^{(j)} T^{(3)} \psi_{1} & =P^{(j)} T^{(3)} \psi_{1}^{\|}+P^{(j)} T^{(3)} \psi_{1}^{\perp} \\
& =\lambda_{1, j} \psi_{1}^{(j)}+P^{(j)} T^{(3)} \psi_{1}^{\perp} .
\end{aligned}
$$

Now insert (3.6) into (3.4). For $j=i$ we have

$$
P^{(i)} T^{(4)} \psi_{0}+P^{(i)} T^{(3)} \psi_{1}^{\perp}=\mathcal{E}_{2} \psi_{0}
$$

Define

$$
\Lambda^{(2, i)}:=P^{(i)}\left(T^{(4)}+T^{(3)} \Xi^{(1, \perp)}\right) P^{(i)}
$$

Then we obtain $\Lambda^{(2, i)} \psi_{0}=\mathcal{E}_{2} \psi_{0}$. By assumption $\Lambda^{(2, i)}$ has only one eigenvalue $\lambda_{2, i}$. Therefore $\mathcal{E}_{2}=\lambda_{2, i}$

For $j \neq i$ we have

$$
P^{(j)} T^{(4)} \psi_{0}+P^{(j)} T^{(3)} \psi_{1}^{\perp}+\lambda_{1, j} \psi_{1}^{(j)}=\lambda_{1, i} P^{(j)} \psi_{1}
$$

because $P^{(j)} \psi_{0}=0$ whenever $j \neq i$. Rearranging terms we finally obtain $\psi_{1}^{(j)}=\Xi^{(1, j)} \psi_{0}$, where we define

$$
\Xi^{(1, j)}:=\left(\lambda_{1, i}-\lambda_{1, j}\right)^{-1} P^{(j)}\left(T^{(4)}+T^{(3)} \Xi^{(1, \perp)}\right) P^{(i)} .
$$

So far no requirement is imposed to $\psi_{1}^{(i)}$.

Now multiply (3.3) by $Q$,

$$
H_{e} \psi_{2}^{\perp}+Q T^{(4)} \psi_{0}+Q T^{(3)} \psi_{1}=\lambda_{1, i} A_{e}^{2} \psi_{1}^{\perp} .
$$


Julio H. Toloza

Since

$$
\begin{aligned}
Q T^{(3)} \psi_{1} & =Q T^{(3)} \psi_{1}^{\perp}+\sum_{l \neq i} Q T^{(3)} \psi_{1}^{(l)}+Q T^{(3)} \psi_{1}^{(i)} \\
& =Q T^{(3)} \Xi^{(1, \perp)} \psi_{0}+\sum_{l \neq i} Q T^{(3)} \Xi^{(1, l)} \psi_{0}+Q T^{(3)} \psi_{1}^{(i)},
\end{aligned}
$$

(3.8) yields

$$
\begin{aligned}
H_{e} \psi_{2}^{\perp}= & -Q T^{(4)} \psi_{0}+\lambda_{1, i} A_{e}^{2} \Xi^{(1, \perp)} \psi_{0} \\
& -Q T^{(3)} \Xi^{(1, \perp)} \psi_{0}-\sum_{l \neq i} Q T^{(3)} \Xi^{(1, l)} \psi_{0}-Q T^{(3)} \psi_{1}^{(i)}
\end{aligned}
$$

From there we obtain

$$
\psi_{2}^{\perp}=\Xi^{(2, \perp)} \psi_{0}+\Xi^{(1, \perp)} \psi_{1}^{(i)}
$$

where

$$
\Xi^{(2, \perp)}:=\left(H_{e}\right)_{\perp}^{-1}\left[\lambda_{1, i} \Xi^{(1, \perp)} A_{e}^{2}-Q T^{(3)}\left(\Xi^{(1, \perp)}+\sum_{l \neq i} \Xi^{(1, l)}\right)-Q T^{(4)}\right] .
$$

Third-order:

$$
H_{e} \psi_{3}+T^{(3)} \psi_{2}+T^{(4)} \psi_{1}+T^{(5)} \psi_{0}=\mathcal{E}_{3} A_{e}^{2} \psi_{0}+\lambda_{2, i} A_{e}^{2} \psi_{1}+\lambda_{1, i} A_{e}^{2} \psi_{2}
$$

Multiply by $P^{(j)}$, rearrange terms, and use (3.5) to obtain

$$
\begin{aligned}
\mathcal{E}_{3} P^{(j)} \psi_{0}= & P^{(j)} T^{(3)} \psi_{2}+P^{(j)} T^{(4)} \psi_{1}+P^{(j)} T^{(5)} \psi_{0}-\lambda_{2, i} \psi_{1}^{(j)}-\lambda_{1, i} \psi_{2}^{(j)} \\
= & P^{(j)} T^{(3)}\left(\psi_{2}^{\perp}+\psi_{2}^{\|}\right)+P^{(j)} T^{(4)}\left(\psi_{1}^{\perp}+\sum_{l \neq i} \psi_{1}^{(l)}+\psi_{1}^{(i)}\right) \\
& +P^{(j)} T^{(5)} \psi_{0}-\lambda_{2, i} \psi_{1}^{(j)}-\lambda_{1, i} \psi_{2}^{(j)} \\
= & P^{(j)} T^{(3)} \psi_{2}^{\perp}+P^{(j)} T^{(4)}\left(\psi_{1}^{\perp}+\sum_{l \neq i} \psi_{1}^{(i)}+\psi_{1}^{(i)}\right) \\
& +P^{(j)} T^{(5)} \psi_{0}-\left(\lambda_{1, i}-\lambda_{1, j}\right) \psi_{2}^{(j)}-\lambda_{2, i} \psi_{1}^{(j)} .
\end{aligned}
$$


For $j=i$ we have

$$
\begin{aligned}
\mathcal{E}_{3} \psi_{0}= & P^{(i)} T^{(3)} \Xi^{(2, \perp)} \psi_{0}+P^{(i)} T^{(4)}\left(\Xi^{(1, \perp)}+\sum_{l \neq i} \Xi^{(1, l)}\right) \psi_{0}+P^{(i)} T^{(5)} \psi_{0} \\
& +P^{(i)} T^{(3)} \Xi^{(1, \perp)} \psi_{1}^{(i)}+P^{(i)} T^{(4)} \psi_{1}^{(i)}-\lambda_{2, i} \psi_{1}^{(i)} .
\end{aligned}
$$

Let us note that

$$
P^{(i)} T^{(4)} \psi^{(i)}+P^{(i)} T^{(3)} \Xi^{(1, \perp)} \psi^{(i)}=\Lambda^{(2, i)} \psi^{(i)}=\lambda_{2, i} \psi^{(i)} .
$$

Thus we obtain $\mathcal{E}_{3} \psi_{0}=\Lambda^{(3, i)} \psi_{0}$, where

$$
\Lambda^{(3, i)}:=P^{(i)}\left[T^{(5)}+T^{(4)}\left(\Xi^{(1, \perp)}+\sum_{l \neq i} \Xi^{(1, l)}\right)+T^{(3)} \Xi^{(2, \perp)}\right] P^{(i)} .
$$

By assumption $\Lambda^{(3, i)}$ has only one eigenvalue $\lambda_{3, i}$ so $\mathcal{E}_{3}=\lambda_{3, i}$.

Now for $j \neq i$ we can rewrite (3.10) as

$$
\begin{aligned}
\left(\lambda_{1, i}-\lambda_{1, j}\right) \psi_{2}^{(j)}= & P^{(j)} T^{(5)} \psi_{0}+P^{(j)} T^{(4)}\left(\Xi^{(1, \perp)}+\sum_{l \neq i} \Xi^{(1, l)}\right) \psi_{0} \\
& +P^{(j)} T^{(3)} \Xi^{(2, \perp)} \psi_{0}-\lambda_{2, i} \Xi^{(1, j)} \psi_{0}+P^{(j)} T^{(3)} \Xi^{(1, \perp)} \psi_{1}^{(i)}+P^{(j)} T^{(4)} \psi_{1}^{(i)} .
\end{aligned}
$$

Now use (3.7) and define

$$
\Xi^{(2, j)}:=\left(\lambda_{1, i}-\lambda_{1, j}\right)^{-1} P^{(j)}\left[T^{(5)}+T^{(4)}\left(\Xi^{(1, \perp)}+\sum_{l \neq i} \Xi^{(1, l)}\right)+T^{(3)} \Xi^{(2, \perp)}-\lambda_{2, i} \Xi^{(1, j)}\right] P^{(i)}
$$

to obtain

$$
\psi_{2}^{(j)}=\Xi^{(2, j)} \psi_{0}+\Xi^{(1, j)} \psi_{1}^{(i)} .
$$

The last step is to multiply (3.9) by Q,

$$
H_{e} \psi_{3}^{\perp}=Q\left(\lambda_{1, i} A_{e}^{2}-T^{(3)}\right) \psi_{2}+Q\left(\lambda_{2, i} A_{e}^{2}-T^{(4)}\right) \psi_{1}-Q T^{(5)} \psi_{0} .
$$


We have

$$
\begin{aligned}
Q\left(\lambda_{1, i} A_{e}^{2}-T^{(3)}\right) \psi_{2}= & Q\left(\lambda_{1, i} A_{e}^{2}-T^{(3)}\right) \psi_{2}^{\perp}+\lambda_{1, i} A_{e}^{2} Q \psi_{2}^{\|}-Q T^{(3)} \sum_{l \neq i} \psi_{2}^{(l)}-Q T^{(3)} \psi_{2}^{(i)} \\
= & Q\left(\lambda_{1, i} A_{e}^{2}-T^{(3)}\right) \Xi^{(2, \perp)} \psi_{0}+Q\left(\lambda_{1, i} A_{e}^{2}-T^{(3)}\right) \Xi^{(1, i)} \psi_{1}^{(i)} \\
& -Q T^{(3)} \sum_{l \neq i} \Xi^{(2, l)} \psi_{0}-Q T^{(3)} \sum_{l \neq i} \Xi^{(1, l)} \psi_{1}^{(i)}-Q T^{(3)} \psi_{2}^{(i)} \\
= & -Q T^{(3)} \psi_{2}^{(i)}+Q\left[\left(\lambda_{1, i} A_{e}^{2}-T^{(3)}\right) \Xi^{(1, \perp)}-\sum_{l \neq i} T^{(3)} \Xi^{(1, l)}\right] \psi_{1}^{(1)} \\
& +Q\left[\left(\lambda_{1, i} A_{e}^{2}-T^{(3)}\right) \Xi^{(2, \perp)}-\sum_{l \neq i} T^{(3)} \Xi^{(2, l)}\right] \psi_{0},
\end{aligned}
$$

and similarly

$$
Q\left(\lambda_{2, i} A_{e}^{2}-T^{(4)}\right) \psi_{1}=Q\left[\left(\lambda_{2, i} A_{e}^{2}-T^{(4)}\right) \Xi^{(1, \perp)}-\sum_{l \neq i} T^{(4)} \Xi^{(1, l)}\right] \psi_{0}-Q T^{(4)} \psi_{1}^{(i)} .
$$

Insert (3.12) and (3.13) in (3.11) and multiply the whole equation by $\left(H_{e}\right)_{\perp}^{-1}$ to obtain

$$
\psi_{3}^{\perp}=\Xi^{(3, \perp)} \psi_{0}+\Xi^{(2, \perp)} \psi_{1}^{(i)}+\Xi^{(1, \perp)} \psi_{2}^{(i)}
$$

with

$$
\begin{aligned}
\Xi^{(3, \perp)}:= & \left(H_{e}\right)_{\perp}^{-1}\left[\left(\lambda_{1, i} \Xi^{(2, \perp)}+\lambda_{2, i} \Xi^{(1, \perp)}\right) A_{e}^{2}-Q T^{(5)}\right. \\
& \left.-Q T^{(4)}\left(\Xi^{(1, \perp)}+\sum_{l \neq i} \Xi^{(1, l)}\right)-Q T^{(5)}\left(\Xi^{(2, \perp)}+\sum_{l \neq i} \Xi^{(2, l)}\right)\right] .
\end{aligned}
$$

As before, one can guess the solution for arbitrary $n$. Let us summarize hypotheses and results:

Proposition 3.2 Define

$$
\Lambda^{(1)}:=P T^{(3)} P
$$


Julio H. Toloza

$$
\Xi^{(1, \perp)}:=-\left(H_{e}\right)_{\perp}^{-1} Q T^{(3)}
$$

Suppose that $\Lambda^{(1)}$ has $k$ distinct eigenvalues $\lambda_{1,1}, \ldots, \lambda_{1, k}$ with eigenspaces $G_{1}, \ldots, G_{k}$. Let $P^{(1)}, \ldots, P^{(k)}$ be the associated projection operators. Given $1 \leq i \leq k$ and $j \neq i$, set

$$
\begin{aligned}
\Lambda^{(2, i)} & :=P^{(i)}\left(T^{(4)}+T^{(3)} \Xi^{(1, \perp)}\right) P^{(i)} \\
\Xi^{(1, j)} & :=\left(\lambda_{1, i}-\lambda_{1, j}\right)^{-1} P^{(j)}\left(T^{(4)}+T^{(3)} \Xi^{(1, \perp)}\right) P^{(i)} \\
\Xi^{(2, \perp)} & :=\left(H_{e}\right)_{\perp}^{-1}\left[\lambda_{1, i} \Xi^{(1, \perp)} A_{e}^{2}-Q T^{(4)}-Q T^{(3)}\left(\Xi^{(1, \perp)}+\sum_{l \neq i} \Xi^{(1, l)}\right)\right] .
\end{aligned}
$$

And then recursively define

$$
\begin{aligned}
\Lambda^{(n, i)}:= & P^{(i)}\left(T^{(n+2)}+\sum_{s=1}^{n-1} T^{(n+2-s)} \Xi^{(s, \perp)}+\sum_{s=1}^{n-2} \sum_{l \neq i} T^{(n+2-s)} \Xi^{(s, l)}\right) P^{(i)} \\
\Xi^{(n-1, j)}:= & \left(\lambda_{1, i}-\lambda_{1, j}\right)^{-1} P^{(j)}\left(T^{(n+2)}+\sum_{s=1}^{n-1} T^{(n+2-s)} \Xi^{(s, \perp)}\right. \\
& \left.+\sum_{s=1}^{n-2} \sum_{l \neq i} T^{(n+2-s)} \Xi^{(s, l)}-\sum_{s=2}^{n-1} \lambda_{s, i} \Xi^{(n-s, j)}\right) P^{(i)} \\
\Xi^{(n, \perp)}:= & \left(H_{e}\right)_{\perp}^{-1}\left[\sum_{s=1}^{n-1} \lambda_{s, i} \Xi^{(n-s, \perp)} A_{e}^{2}-Q T^{(n+2)}-\sum_{s=1}^{n-1} Q T^{(s+2)}\left(\Xi^{(n-s, \perp)}+\sum_{l \neq i} \Xi^{(n-s, l)}\right)\right]
\end{aligned}
$$

where $\lambda_{s, i}$ is, by assumption, the unique eigenvalue of $\Lambda^{(s, i)}$ when $s \geq 2$.

Let $\mathcal{E}_{n}, \psi_{n}$ be the $R$-S coefficients. Then $\mathcal{E}_{1}$ has to be equal to one of the eigenvalues of $\Lambda^{(1)}$, let us say $\mathcal{E}_{1}=\lambda_{1, i}$. Consequently, $\psi_{0} \in G_{i}$ and

$$
\begin{aligned}
\mathcal{E}_{n} & =\lambda_{n, i}, \\
\psi_{n-1}^{(j)} & =\Xi^{(n-1, j)} \psi_{0}+\sum_{s=1}^{n-1} \Xi^{(n-s-1, j)} \psi_{s}^{(i)} \\
\psi_{n}^{\perp} & =\Xi^{(n, \perp)} \psi_{0}+\sum_{s=1}^{n-1} \Xi^{(n-s, \perp)} \psi_{s}^{(i)}
\end{aligned}
$$


Julio H. Toloza

$$
\psi_{n}=\psi_{n}^{\perp}+\sum_{j \neq i} \psi_{n}^{(j)}+\psi_{n}^{(i)}
$$

The vectors $\psi_{1}^{(i)}, \ldots, \psi_{n}^{(i)}$ are arbitrarily chosen from $G_{i}$.

Proof. Use mathematical induction. Because of the discussion above, we only have to prove the inductive step. Thus, let us assume that $\mathcal{E}_{m}, \psi_{m-1}^{(j)}$ and $\psi_{m}^{\perp}$ are given by (3.14)-(3.16), for $m=2, \ldots, n$. Let us compute $\mathcal{E}_{n+1}, \psi_{n}^{(j)}$ and $\psi_{n+1}^{\perp}$. The $(n+1)$-st order equation is

$$
H_{e} \psi_{n+1}+\sum_{p=0}^{n} T^{(n+3-p)} \psi_{p}=\sum_{s=0}^{n} \mathcal{E}_{n+1-s} A_{e}^{2} \psi_{s}
$$

We have

$$
\begin{aligned}
& \sum_{p=0}^{n} T^{(n+3-p)} \psi_{p}=T^{(n+3)} \psi_{0}+\sum_{p=1}^{n} T^{(n+3-p)} \psi_{p}^{\perp}+\sum_{p=1}^{n} T^{(n+3-p)} \sum_{l \neq i} \psi_{p}^{(l)}+\sum_{p=1}^{n} T^{(n+3-p)} \psi_{p}^{(i)} \\
& =T^{(n+3)} \psi_{0}+T^{(n+2)} \Xi^{(1, \perp)} \psi_{0}+\sum_{p=2}^{n} T^{(n+3-p)}\left(\Xi^{(p, \perp)} \psi_{0}+\sum_{s=1}^{p-1} \Xi^{(p-s, \perp)} \psi_{s}^{(i)}\right) \\
& +\sum_{l \neq i} T^{(n+2)} \Xi^{(1, l)} \psi_{0}+\sum_{p=2}^{n-1} \sum_{l \neq i} T^{(n+3-p)}\left(\Xi^{(p, l)} \psi_{0}+\sum_{s=1}^{p-1} \Xi^{(p-s, l)} \psi_{s}^{(i)}\right) \\
& +\sum_{l \neq i} T^{(3)} \psi_{n}^{(l)}+\sum_{p=1}^{n} T^{(n+3-p)} \psi_{p}^{(i)} \\
& =\left(T^{(n+3)}+\sum_{p=1}^{n} T^{(n+3-p)} \Xi^{(p, \perp)}+\sum_{p=1}^{n-1} \sum_{l \neq i} T^{(n+3-p)} \Xi^{(p, l)}\right) \psi_{0} \\
& +\sum_{s=1}^{n-1} \sum_{p=s+1}^{n} T^{(n+3-p)} \Xi^{(p-s, \perp)} \psi_{s}^{(i)}+\sum_{s=1}^{n-2} \sum_{p=s+1}^{n-1} \sum_{l \neq i} T^{(n+3-p)} \Xi^{(p-s, l)} \psi_{s}^{(i)} \\
& +\sum_{l \neq i} T^{(3)} \psi_{n}^{(l)}+\sum_{s=1}^{n} T^{(n+3-s)} \psi_{s}^{(i)} \\
& =\left(T^{(n+3)}+\sum_{p=1}^{n} T^{(n+3-p)} \Xi^{(p, \perp)}+\sum_{p=1}^{n-1} \sum_{l \neq i} T^{(n+3-p)} \Xi^{(p, l)}\right) \psi_{0} \\
& +\sum_{s=1}^{n-1} \sum_{m=1}^{n-s} T^{(n+3-s-m)} \Xi^{(m, \perp)} \psi_{s}^{(i)}+\sum_{s=1}^{n-2} \sum_{m=1}^{n-1-s} \sum_{l \neq i} T^{(n+3-s-m)} \Xi^{(m, l)} \psi_{s}^{(i)}
\end{aligned}
$$




$$
+\sum_{l \neq i} T^{(3)} \psi_{n}^{(l)}+\sum_{s=1}^{n} T^{(n+3-s)} \psi_{s}^{(i)}
$$

where we use that $\sum_{p=1}^{r} \sum_{s=1}^{p-1} F_{s p}=\sum_{s=1}^{r-1} \sum_{p=s+1}^{r} F_{s p}$ and then we change index $p \rightarrow m=$ $p-s$

Let us multiply (3.17) by $P^{(i)}$. Since $P^{(i)} H_{e}=0$ and $P^{(i)} A_{e}^{2}=A_{e}^{2} P^{(i)}=P^{(i)}$, we obtain

$$
\sum_{p=0}^{n} P^{(i)} T^{(n+3-p)} \psi_{p}=\mathcal{E}_{n+1} \psi_{0}+\sum_{s=1}^{n} \lambda_{n+1-s, i} \psi_{s}^{(i)} .
$$

The left-hand side can be written as

$$
\begin{aligned}
\sum_{p=0}^{n} P^{(i)} T^{(n+3-p)} \psi_{p}= & P^{(i)}\left(T^{(n+3)}+\sum_{p=1}^{n} T^{(n+3-p)} \Xi^{(p, \perp)}+\sum_{p=1}^{n-1} \sum_{l \neq i} T^{(n+3-p)} \Xi^{(p, l)}\right) \psi_{0} \\
& +\sum_{s=1}^{n-2} P^{(i)}\left(T^{(n+3-s)}+\sum_{m=1}^{n-s} T^{(n+3-s-m)} \Xi^{(m, \perp)}\right. \\
& \left.+\sum_{m=1}^{n-1-s} \sum_{l \neq i} T^{(n+3-s-m)} \Xi^{(m, l)}\right) \psi_{s}^{(i)} \\
& +P^{(i)}\left(T^{(3)} \Xi^{(1, \perp)}+T^{(4)}\right) \psi_{n-1}^{(i)}+\sum_{l \neq i} P^{(i)} T^{(3)} \psi_{n}^{(l)}+P^{(i)} T^{(3)} \psi_{n}^{(i)} .
\end{aligned}
$$

By the argument that leads to (3.5), we know that $\sum_{l \neq i} P^{(i)} T^{(3)} \psi_{n}^{(l)}=0$. Also $\psi_{s}^{(i)}=P^{(i)} \psi_{s}^{(i)}$.

Then

$$
\sum_{p=0}^{n} P^{(i)} T^{(n+3-p)} \psi_{p}=\Lambda^{(n+1, i)} \psi_{0}+\sum_{s=1}^{n} \Lambda^{(n+1-s, i)} \psi_{s}^{(i)} .
$$

Inserting (3.19) into (3.18) we conclude

$$
\Lambda^{(n+1, i)} \psi_{0}=\mathcal{E}_{n+1} \psi_{0}
$$

Now let us multiply $(3.17)$ by $P^{(j)}$ for $j \neq i$. Since $P^{(j)} \psi_{0}=0$, we have

$$
\lambda_{1, i} \psi_{n}^{(j)}=\sum_{p=0}^{n} P^{(j)} T^{(n+3-p)} \psi_{p}-\sum_{s=1}^{n-1} \lambda_{n+1-s, i} \psi_{s}^{(j)} .
$$


The right-hand side can be manipulated in the same way as before. The result is

$$
\begin{aligned}
\sum_{p=0}^{n} P^{(i)} T^{(n+3-p)} \psi_{p}- & \sum_{s=1}^{n-1} \lambda_{n+1-s, i} \psi_{s}^{(j)} \\
& =\left(\lambda_{1, i}-\lambda_{1, j}\right) \Xi^{(n, j)} \psi_{0}+\sum_{s=1}^{n-1}\left(\lambda_{1, i}-\lambda_{1, j}\right) \Xi^{(n-s, j)} \psi_{s}^{(i)}+\sum_{l=1}^{k} P^{(j)} T^{(3)} \psi_{n}^{(l)} .
\end{aligned}
$$

As proven in (3.5), the last term above is equal to $\lambda_{1, j} \psi_{n}^{(j)}$. Thus (3.20) leads to

$$
\psi_{n}^{(j)}=\Xi^{(n, j)} \psi_{0}+\sum_{s=1}^{n-1} \Xi^{(n-s, j)} \psi_{s}^{(i)} .
$$

Finally, multiply (3.17) by $Q$,

$$
H_{e} \psi_{n+1}^{\perp}=\sum_{p=1}^{n} \lambda_{n+1-p, i} A_{e}^{2} \psi_{p}^{\perp}-\sum_{p=0}^{n} Q T^{(n+3-p)} \psi_{p} .
$$

For the first term we have

$$
\begin{aligned}
\sum_{p=1}^{n} \lambda_{n+1-p, i} A_{e}^{2} \psi_{p}^{\perp} & =\sum_{s=1}^{n} \lambda_{n+1-s, i} A_{e}^{2} \Xi^{(s, \perp)} \psi_{0}+\sum_{p=2}^{n} \sum_{s=1}^{p-1} \lambda_{n+1-p, i} A_{e}^{2} \Xi^{(p-s, \perp)} \psi_{s}^{(i)} \\
& =\sum_{s=1}^{n} \lambda_{n+1-s, i} A_{e}^{2} \Xi^{(s, \perp)} \psi_{0}+\sum_{s=1}^{n-1} \sum_{m=1}^{n-s} \lambda_{n+1-s-m, i} A_{e}^{2} \Xi^{(m, \perp)} \psi_{s}^{(i)},
\end{aligned}
$$

and for the second one

$$
\begin{aligned}
\sum_{p=0}^{n} Q T^{(n+3-p)} \psi_{p}= & Q\left(T^{(n+3)}+\sum_{p=1}^{n} T^{(n+3-p)} \Xi^{(p, \perp)}+\sum_{p=1}^{n-1} \sum_{l \neq i} T^{(n+3-p)} \Xi^{(p, l)}\right) \psi_{0} \\
& +\sum_{s=1}^{n-2} Q\left(T^{(n+3-s)}+\sum_{m=1}^{n-s} T^{(n+3-s-m)} \Xi^{(m, \perp)}\right. \\
& \left.+\sum_{m=1}^{n-1-s} \sum_{l \neq i} T^{(n+3-s-m)} \Xi^{(m, l)}\right) \psi_{s}^{(i)} \\
& +Q\left(T^{(3)} \Xi^{(1, \perp)}+T^{(4)}\right) \psi_{n-1}^{(i)}+\sum_{l \neq i} Q T^{(3)} \psi_{n}^{(l)}+Q T^{(3)} \psi_{n}^{(i)} .
\end{aligned}
$$


Then insert these expressions into (3.21). After multiplying the whole equation by $\left(H_{e}\right)_{\perp}^{-1}$ we obtain the desired result.

As before, we set $\psi_{n}^{(i)}=0$ for all $n=1,2, \ldots$. Consequently, $\psi_{n}$ will be orthogonal to $\psi_{0}$ and

$$
\psi_{n}=\left(\Xi^{(n, \perp)}+\sum_{l \neq i} \Xi^{(n, l)}\right) \psi_{0} .
$$

The following expressions will be useful later:

$$
\begin{aligned}
\Lambda^{(n, i)} \psi_{0}= & P^{(i)} T^{(n+2)} \psi_{0}+\sum_{s=1}^{n-2} P^{(i)} T^{(n+2-s)} \psi_{s}+P^{(i)} T^{(3)} \psi_{n-1}^{\perp} \\
\psi_{n}^{\perp}= & \left(H_{e}\right)_{\perp}^{-1}\left[\sum_{s=1}^{n-1} \mathcal{E}_{s} A_{e}^{2} \psi_{n-s}^{\perp}-Q T^{(n+2)} \psi_{0}-\sum_{s=1}^{n-1} Q T^{(s+2)} \psi_{n-s}\right] \\
\psi_{n-1}^{(j)}= & \left(\lambda_{1, i}-\lambda_{1, j}\right)^{-1}\left(P^{(j)} T^{(n+2)} \psi_{0}+\sum_{s=1}^{n-2} P^{(j)} T^{(n+2-s)} \psi_{s}\right. \\
& \left.+P^{(j)} T^{(3)} P_{|j| \leq a+3(n-1)} \psi_{n-1}^{\perp}-\sum_{s=2}^{n-1} \mathcal{E}_{s} \psi_{n-s}^{(j)}\right) .
\end{aligned}
$$

Next, let us estimate the growth of these coefficients. Since $\mathcal{E}_{n} \psi_{0}=\Lambda^{(n, i)} \psi_{0}$,

$$
\begin{aligned}
\left|\mathcal{E}_{n}\right| & =\left|\left\langle\psi_{0}, \Lambda^{(n, i)} \psi_{0}\right\rangle\right| \\
& \leq\left|\left\langle\psi_{0}, P^{(i)} T^{(n+2)} \psi_{0}\right\rangle\right|+\sum_{s=1}^{n-2}\left|\left\langle\psi_{0}, P^{(i)} T^{(n+2-s)} \psi_{s}\right\rangle\right|+\left|\left\langle\psi_{0}, P^{(i)} T^{(3)} \psi_{n-1}^{\perp}\right\rangle\right| \\
& \leq\left\|T^{(n+2)} P_{|\alpha| \leq a}\right\|+\sum_{s=1}^{n-2}\left|\left\langle T^{(n+2-s)} \psi_{0}, \psi_{s}\right\rangle\right|+\left|\left\langle T^{(3)} \psi_{0}, \psi_{n-1}^{\perp}\right\rangle\right| \\
& \leq\left\|T^{(n+2)} P_{|\alpha| \leq a}\right\|+\sum_{s=1}^{n-2}\left\|T^{(n+2-s)} P_{|\alpha| \leq a}\right\|\left\|\psi_{s}\right\|+\left\|T^{(3)} P_{|\alpha| \leq a}\right\|\left\|\psi_{n-1}^{\perp}\right\| \\
& =\sum_{s=2}^{n}\left\|T^{(s+2)} P_{|\alpha| \leq a}\right\|\left\|\psi_{n-s}\right\|+\left\|T^{(3)} P_{|\alpha| \leq a}\right\|\left\|\psi_{n-1}^{\perp}\right\| .
\end{aligned}
$$

This calculation follows from (3.22), the self-adjointness of $T^{(l)}$, and Lemma 2.1. 
From the definition of $H_{e}$, it is straightforward to see that $\left\|\left(H_{e}\right)_{\perp}^{-1}\right\|=1$. Also, $\left\|A_{e}\right\|=1$.

Thus, from (3.23) we have

$$
\begin{aligned}
\left\|\psi_{n}^{\perp}\right\| & \leq \sum_{s=1}^{n-1}\left|\mathcal{E}_{s}\right|\left\|\psi_{n-s}^{\perp}\right\|+\left\|T^{(n+2)} P_{|\alpha| \leq a}\right\|\left\|\psi_{0}\right\|+\sum_{s=1}^{n-1}\left\|T^{(s+2)} P_{|\alpha| \leq a+3(n-s)}\right\|\left\|\psi_{n-s}\right\| \\
& =\sum_{s=1}^{n-1}\left|\mathcal{E}_{s}\right|\left\|\psi_{n-s}^{\perp}\right\|+\sum_{s=1}^{n}\left\|T^{(s+2)} P_{|\alpha| \leq a+3(n-s)}\right\|\left\|\psi_{n-s}\right\|
\end{aligned}
$$

Finally let us consider (3.24)

$$
\begin{aligned}
\left\|\psi_{n-1}^{(j)}\right\| \leq & \left|\lambda_{1, i}-\lambda_{1, j}\right|^{-1}\left(\left\|T^{(n+2)} P_{|\alpha| \leq a}\right\|\left\|\psi_{0}\right\|+\sum_{s=1}^{n-2}\left\|P^{(j)} T^{(n+2-s)}\right\|\left\|\psi_{s}\right\|\right. \\
& \left.+\left\|P^{(j)} T^{(3)}\right\|\left\|\psi_{n-1}^{\perp}\right\|+\sum_{s=2}^{n-1}\left|\mathcal{E}_{s}\right|\left\|\psi_{n-s}^{(j)}\right\|\right) .
\end{aligned}
$$

Set $C_{7}:=\min _{j \neq i}\left|\lambda_{1, i}-\lambda_{1, j}\right|^{-1}$. Also, let us notice that $\left\|P^{(j)} T^{(n+2-s)}\right\|=\left\|T^{(n+2-s)} P^{(j)}\right\|=$ $\left\|T^{(n+2-s)} P_{|\alpha| \leq a} P^{(j)}\right\| \leq\left\|T^{(n+2-s)} P_{|\alpha| \leq a}\right\|$. Thus,

$$
\left\|\psi_{n-1}^{(j)}\right\| \leq C_{7} \sum_{s=2}^{n-1}\left|\mathcal{E}_{s}\right|\left\|\psi_{n-s}^{(j)}\right\|+C_{7} \sum_{s=2}^{n}\left\|T^{(s+2)} P_{|\alpha| \leq a}\right\|\left\|\psi_{n-s}\right\|+C_{7}\left\|T^{(3)} P_{|\alpha| \leq a}\right\|\left\|\psi_{n-1}^{\perp}\right\| .
$$

These inequalities will allow us to obtain upper bounds for the growth of R-S coefficients. In the following theorem we make use of the Lemmas 2.3 and 2.5.

Theorem 3.2 Let $k$ be the number of subspaces as defined in Proposition 3.2. Define $b_{1}:=$ $C_{3}\left[k C_{6}+(2+a)^{\frac{1}{2}}\right], b_{2}:=8 C_{7}\left[b_{1} C_{4}+C_{3}(2+a)^{\frac{1}{2}}+k C_{3} C_{6}\right]$ and $b_{3}:=b_{1} C_{4}+C_{3} C_{5}[1+$ $\left.b_{2}(k-1)\right]$. Then for any $b \geq \max \left\{b_{1}, b_{2}, b_{3}, 1\right\}$ and for $n=1,2, \ldots$,

$$
\begin{aligned}
\left|\mathcal{E}_{n}\right| & \leq b_{1} \kappa^{3 n} b^{n-2}[(a+n) !]^{\frac{1}{2}} \\
\left\|\psi_{n-1}^{(l)}\right\| & \leq b_{2} \kappa^{3(n-1)} b^{n-2}[(a+n) !]^{\frac{1}{2}}
\end{aligned}
$$


Julio H. Toloza

$$
\left\|\psi_{n}^{\perp}\right\| \leq b_{3} \kappa^{3 n} b^{n-2}[(1+a+n) !]^{\frac{1}{2}} .
$$

Proof. Assume the estimates are true for $s=1, \ldots, n-1$. This implies that

$$
\left\|\psi_{s}\right\| \leq\left[b_{3}+b_{2}(k-1)\right] \kappa^{3 s} b^{s-1}[(1+a+s) !]^{\frac{1}{2}} \leq \kappa^{3 s} k b^{s}[(1+a+s) !]^{\frac{1}{2}}
$$

for $s \leq n-2$. We shall use the second inequality in (3.31) to prove (3.28) and (3.29), and the first one to prove (3.30).

Let us start showing (3.28). Applying Lemmas 2.3 and 2.5, statement 2, we obtain

$$
\begin{aligned}
\sum_{s=2}^{n}\left\|T^{(s+2)} P_{|\alpha| \leq a}\right\|\left\|\psi_{n-s}\right\| & \leq C_{3} k \sum_{s=2}^{n} \kappa^{\frac{s}{2}}\left[\frac{(1+a+s) !}{(1+a) !}\right]^{\frac{1}{2}} \kappa^{3(n-s)} b^{n-s}[(1+a+n-s) !]^{\frac{1}{2}} \\
& \leq C_{3} k \kappa^{3 n} b^{n-2}[(a+n) !]^{\frac{1}{2}} \sum_{s=2}^{n} \kappa^{-\frac{5 s}{2}}\left[\frac{(1+a+s) !(1+a+n-s) !}{(1+a) !(a+n) !}\right]^{\frac{1}{2}} \\
& \leq k C_{3} C_{6} \kappa^{3 n} b^{n-2}[(a+n) !]^{\frac{1}{2}}
\end{aligned}
$$

Thus, (3.25) yields

$$
\begin{aligned}
\left|\mathcal{E}_{n}\right| & \leq k C_{3} C_{6} \kappa^{3 n} b^{n-2}[(a+n) !]^{\frac{1}{2}}+C_{3} \kappa^{3(n-1)} b_{3} b^{n-3} \kappa^{\frac{1}{2}}(2+a)^{\frac{1}{2}}[(a+n) !]^{\frac{1}{2}} \\
& \leq k C_{3} C_{6} \kappa^{3 n} b^{n-2}[(a+n) !]^{\frac{1}{2}}+C_{3}(2+a)^{\frac{1}{2}} \kappa^{3 n} b^{n-2}[(a+n) !]^{\frac{1}{2}} \\
& \leq b_{1} \kappa^{3 n} b^{n-2}[(a+n) !]^{\frac{1}{2}}
\end{aligned}
$$

which completes the proof of (3.28).

To prove (3.29) we start from (3.27) and proceed in the same fashion $\left\|\psi_{n-1}^{(j)}\right\| \leq C_{7} \kappa^{3 n} b_{1} b_{2} b^{n-3} \sum_{s=2}^{n-1}[(a+s) !(1+a+n-s) !]^{\frac{1}{2}}+C_{3} C_{7} \kappa^{3 n} b_{3} b^{n-3}(2+a)^{\frac{1}{2}}[(a+n) !]^{\frac{1}{2}}$ 


$$
\begin{aligned}
& +C_{3} C_{7} k \kappa^{3 n} b^{n-2} \sum_{s=2}^{n} \kappa^{-\frac{5 s}{2}}\left[\frac{(1+a+s) !(1+a+n-s) !}{(1+a) !}\right]^{\frac{1}{2}} \\
& \leq C_{7} b_{1} \kappa^{3 n} b^{n-2}[(a+n) !]^{\frac{1}{2}} \sum_{m=1}^{n-2}\left[\frac{(1+a+m) !(a+n-m) !}{(a+n) !}\right]^{\frac{1}{2}} \\
& +C_{3} C_{7}(2+a)^{\frac{1}{2}} \kappa^{3 n} b^{n-2}[(a+n) !]^{\frac{1}{2}} \\
& +C_{3} C_{7} k \kappa^{3 n} b^{n-2}[(a+n) !]^{\frac{1}{2}} \sum_{s=2}^{n} \kappa^{-\frac{5 s}{2}}\left[\frac{(1+a+s) !(1+a+n-s) !}{(1+a) !(a+n) !}\right]^{\frac{1}{2}}
\end{aligned}
$$

where we have changed index $s \rightarrow m=s-1$ in the first term. From this and statements 1 and 3 of Lemma 2.5, we obtain

$$
\begin{aligned}
\left\|\psi_{n-1}^{(j)}\right\| & \leq 8 C_{7}\left[b_{1} C_{4}+C_{3}(2+a)^{\frac{1}{2}}+k C_{3} C_{6}\right] \kappa^{3(n-1)} b^{n-2}[(a+n) !]^{\frac{1}{2}} \\
& =b_{2} \kappa^{3(n-1)} b^{n-2}[(a+n) !]^{\frac{1}{2}}
\end{aligned}
$$

so (3.29) is done. Consequently, (3.31) must be valid for $s=n-1$.

Finally let us show (3.30). Note that the first term of (3.26) is bounded like the first term of (3.27). Applying statement 2 of Lemma 2.5, it follows that

$$
\begin{aligned}
\left\|\psi_{n}^{\perp}\right\| \leq & b_{1} b_{3} \kappa^{3 n} b^{n-3} C_{6}[(a+n) !]^{\frac{1}{2}} \\
& +C_{3}\left[1+b_{2}(k-1)\right] \kappa^{3 n} b^{n-2}[(1+a+n) !]^{\frac{1}{2}} \sum_{s=1}^{n} \kappa^{-\frac{5 s}{2}}\left[\frac{(1+a+3 n-2 s) !(1+a+n-s) !}{(1+a+3 n-3 s) !(1+a+n) !}\right]^{\frac{1}{2}} \\
\leq & b_{1} C_{6} \kappa^{3 n} b^{n-2}[(1+a+n) !]^{\frac{1}{2}}+C_{3}\left[1+b_{2}(k-1)\right] C_{5} \kappa^{3 n} b^{n-2}[(1+a+n) !]^{\frac{1}{2}} \\
= & b_{3} \kappa^{3 n} b^{n-2}[(1+a+n) !]^{\frac{1}{2}}
\end{aligned}
$$

\section{Corollary 3.1}

$$
\left|\mathcal{E}_{n}\right| \leq \kappa^{3 n} b^{n-1}[(a+n) !]^{\frac{1}{2}}
$$




$$
\left\|\psi_{n}\right\| \leq \kappa^{3 n} k b^{n}[(1+a+n) !]^{\frac{1}{2}}
$$

For the case where degeneracy is partly broken only up to second order, one needs to define certain operators $\Lambda^{\left(n, i_{1}, i_{2}\right)}, \Xi^{\left(n-2, i_{1}, i_{2}\right)}, \Xi^{(n, \perp)}$ for $n \geq 3$, in addition to those already defined in the last subsection. Now $\psi_{0}$ would be required to lie in a certain subspace $G_{i_{1}, 1_{2}} \in G_{i_{1}} \in G$, and one would be able to determine $\psi_{n}$ module an arbitrary component in $G_{i_{1}, 1_{2}}$. This scheme may be extended to the general case. But the complexity of the set of equations that recursively defines those operators rapidly becomes wild. For that reason, we do not go further. We assume instead that, in general,

$$
\begin{aligned}
\left|\mathcal{E}_{n}\right| & \leq \kappa^{3 n} b^{n+w}[(1+a+n) !]^{\frac{1}{2}} \\
\left\|\psi_{n}\right\| & \leq \kappa^{3 n} b^{n+w}[(1+a+n) !]^{\frac{1}{2}}
\end{aligned}
$$

for some positive integer $w$, which may depend on where degeneracy splits. 


\section{Chapter 4}

\section{The main estimate}

The upper bounds for $\left|\mathcal{E}_{n}\right|$ and $\left\|\psi_{n}\right\|$ will allow us to estimate the error made in the Schrödinger equation when truncated series are inserted on it. Here we basically follow the technique developed by Hagedorn and Joye in [8]. Concretely, for $N \geq 1$ define

$$
E_{N}:=e+\sum_{n=1}^{N-1} \hbar^{\frac{n}{2}} \mathcal{E}_{n} \quad \Psi_{N}(x):=\psi_{0}(x)+\sum_{n=1}^{N-1} \hbar^{\frac{n}{2}} \psi_{n}(x) .
$$

These are the truncations at order $N$ of the R-S series. We define

$$
\begin{aligned}
\xi_{N}(x) & :=A_{e}\left[H_{0}+W(\hbar ; x)-E_{N}\right] A_{e} \Psi_{N}(x) \\
& =\left[H_{e}+A_{e} W(\hbar ; x) A_{e}-\sum_{j=1}^{N-1} \hbar^{\frac{j}{2}} \mathcal{E}_{j} A_{e}^{2}\right] \sum_{m=0}^{N-1} \hbar^{\frac{m}{2}} \psi_{m}(x) .
\end{aligned}
$$

We call $\xi_{N}(x)$ the two-side error function since it is the difference between both sides of the Schrödinger equation when exact eigenvalues and eigenfunctions are replaced by truncated series. It can be portrayed in a more suitable way through a number of cancellations. The 
calculation is outlined in the Appendix. The result is

$$
\xi_{N}(x)=\sum_{n=0}^{N-1} \hbar^{\frac{n}{2}} A_{e} W^{[N-n+1]}(\hbar ; x) A_{e} \psi_{n}(x)-\sum_{n=N}^{2 N-2} \hbar^{\frac{n}{2}} \sum_{j=n-N+1}^{N-1} \mathcal{E}_{j} A_{e}^{2} \psi_{m-j}(x) .
$$

Here $W^{[j]}(\hbar ; x)$ is the tail of the Taylor series of $V(\hbar ; x)$ :

$$
W^{[j]}(\hbar ; x)=V(\hbar ; x)-\sum_{l=2}^{j} \hbar^{\frac{l-2}{2}} \sum_{|\alpha|=l} \frac{D^{\alpha} V(0)}{\alpha !} x^{\alpha}=\hbar^{\frac{j-1}{2}} \sum_{|\alpha|=j+1} \frac{D^{\alpha} V\left(\zeta_{j}\right)}{\alpha !} x^{\alpha}
$$

where $\zeta_{j}=\zeta_{j}(x)=\Theta_{j} x$ with $\Theta_{j} \in(0,1)$, as the Taylor theorem states. So we have

$$
\xi_{N}(x)=\hbar^{\frac{N}{2}} \sum_{n=0}^{N-1} \sum_{|\alpha|=N-n+2} \frac{D^{\alpha} V\left(\zeta_{n}\right)}{\alpha !} A_{e} x^{\alpha} A_{e} \psi_{n}(x)-\sum_{n=N}^{2 N-2} \hbar^{\frac{n}{2}} \sum_{l=n-N+1}^{N-1} \mathcal{E}_{l} A_{e}^{2} \psi_{n-l}(x) .
$$

Our main result in the next chapter relies on an upper bound of the $L^{2}$-norm of $(H-$ $\left.E_{N}\right) A_{e} \psi_{N}=A_{e}^{-1} \xi_{N}$. Note that, for each $N \geq 2, \xi_{N}$ is in the domain of the unbounded operator $A_{e}^{-1}$. This estimate on the two-side error function is stated as follows:

Theorem 4.1 There are positive constants $A, B$ and $N_{0}$ so that

$$
\left\|A_{e}^{-1} \xi_{N}(x)\right\| \leq \sum_{n=N}^{2 N} A B^{N} \hbar^{\frac{N}{2}}[(2+a+n) !]^{\frac{1}{2}}
$$

whenever $N_{0} \leq N$ and $\hbar \leq 1$.

To estimate the norm of $A_{e}^{-1} \xi_{N}$, we first set a suitable closed region around the bottom of the potential well. Then we compute that norm inside and outside of that region. Most of the work is involved in the outside estimate, which requires control on the growth of derivatives of $V(x)$ far away from the minimum of $V(x)$. For that reason we shall summarize it as a separate lemma. Here the hypothesis H5 becomes crucial. 
For $R>0$, let us define

$$
\chi_{R}(x)= \begin{cases}1 & \text { if } \sum_{i}^{d} \omega_{i} x_{i}^{2} \leq R^{2} \\ 0 & \text { otherwise. }\end{cases}
$$

Lemma 4.1 Set $R=\sqrt{6 N+2 a+d-4}$. Given a multi-index $\alpha$, with $|\alpha| \geq 2$, and $n=$ $0, \ldots, N-1$, there exists certain constants $C_{8}$ and $C_{9}$ such that

$$
\left\|\frac{\delta^{|\alpha|}}{\alpha !} D^{\alpha} V\left(\zeta_{n}\right) x^{\alpha^{\prime}}\left(1-\chi_{R}\right) P_{|\beta| \leq a+3 n+1}\right\| \leq C_{8} C_{9}^{\frac{3 n+2+a}{2}} \frac{(3 n+a+d)^{\frac{d-1}{2}}}{\left(1-\frac{\tau}{\omega_{0}}\right)^{\frac{|\alpha|}{2}}}\left[\frac{(3 n+|\alpha|+\llbracket d / 2 \rrbracket+a) !}{(3 n+a) !}\right]^{\frac{1}{2}}
$$

where $\left|\alpha^{\prime}\right|=|\alpha|-1, \omega_{0}=\min \left\{\omega_{1}, \ldots, \omega_{d}\right\}$, and $\llbracket J \rrbracket$ stands for the largest integer less than or equal to $J$.

Proof. Since $\left|\zeta_{n}\right| \leq|x|$, the first part of Lemma 2.3 implies

$$
\frac{\delta^{|\alpha|}}{\alpha !}\left|D^{\alpha} V\left(\zeta_{n}\right)\right| \leq C_{0} \exp \left(2 \tau x^{2}\right)
$$

Let us consider an eigenfunction $\Phi_{\beta}(x)$ of $H_{0}$. We have

$$
\begin{aligned}
\left\|\frac{\delta^{|\alpha|}}{\alpha !} D^{\alpha} V(\zeta) x^{\alpha^{\prime}}\left(1-\chi_{R}\right) \Phi_{\beta}(x)\right\|^{2} & =\int_{\mathbb{R}^{d}}\left|\frac{\delta^{|\alpha|}}{\alpha !} D^{\alpha} V(\zeta)\right|^{2} x^{2 \alpha^{\prime}}\left|\Phi_{\beta}(x)\right|^{2}\left[1-\chi_{R}(x)\right] d^{d} x \\
& \leq C_{0}^{2} \int_{\mathbb{R}^{d}} e^{4 \tau x^{2}} x^{2 \alpha^{\prime}}\left|\Phi_{\beta}(x)\right|^{2}\left[1-\chi_{R}(x)\right] d^{d} x
\end{aligned}
$$

where we have dropped the index $n$ in $\zeta_{n}$. Now change variables $x_{i} \rightarrow y_{i}=\sqrt{\omega_{i}} x_{i}$ to get

$$
\begin{aligned}
\| \frac{\delta^{|\alpha|}}{\alpha !} D^{\alpha} V(\zeta) x^{\alpha^{\prime}}\left(1-\chi_{R}\right) \Phi_{\beta}(x) & \|^{2} \\
& \leq C_{0}^{2}\left(\prod_{i=1}^{d} \omega_{i}^{-\alpha_{i}^{\prime}-\frac{1}{2}}\right) \int_{\mathbb{R}^{d}} e^{\sum_{i} 4 \frac{\tau}{\omega_{i}} y_{i}^{2}} y^{2 \alpha^{\prime}}\left|\Phi_{\beta}(y)\right|^{2}\left[1-\chi_{R}(y)\right] d^{d} y \\
& \leq C_{0}^{2}\left(\prod_{i=1}^{d} \omega_{i}^{-\alpha_{i}^{\prime}-\frac{1}{2}}\right) \int_{\mathbb{R}^{d}} e^{4 \frac{\tau}{\omega_{0}} y^{2}} y^{2 \alpha^{\prime}}\left|\Phi_{\beta}(y)\right|^{2}\left[1-\chi_{R}(y)\right] d^{d} y
\end{aligned}
$$




$$
=D_{1}^{2}\left\|e^{2 \frac{\tau}{\omega_{0}} y^{2}} y^{\alpha^{\prime}}\left(1-\chi_{R}\right) \Phi_{\beta}(y)\right\|^{2}
$$

where $D_{1}$ is defined in the obvious way. In the new variables

$$
\chi_{R}(y)=\left\{\begin{array}{cc}
1 & \text { if } y^{2} \leq R^{2} \\
0 & \text { otherwise }
\end{array}\right.
$$

Using the new variables in $(2.4)$, we see that $\Phi_{\beta}(y)$ is an eigenfunction of the normalized harmonic oscillator operator

$$
H_{0}^{\prime}=-\frac{1}{2} \Delta_{y}+\frac{1}{2} y^{2}
$$

with energy $e_{\beta}=|\beta|+d / 2$. For $d \geq 2$ this operator is equal to

$$
H_{0}^{\prime}=\frac{1}{2}\left(-\frac{\partial^{2}}{\partial r^{2}}-\frac{d-1}{r} \frac{\partial}{\partial r}+\frac{\mathcal{L}^{2}}{r^{2}}+r^{2}\right)
$$

in spherical coordinates, where $\mathcal{L}^{2}$ is the angular momentum operator defined on $S^{d-1}$. The eigenvalues now read $e=2 n+q+d / 2$ and the eigenfunctions are

$$
\Psi_{k, q, \nu}(r, \omega)=\left[\frac{2 k !}{\Gamma\left(k+q+\frac{d}{2}\right)}\right]^{\frac{1}{2}} r^{q} L_{k}^{q+\frac{d}{2}-1}\left(r^{2}\right) \exp \left(-\frac{r^{2}}{2}\right) Y_{q, \nu}(\omega) .
$$

Here $Y_{q, \nu}(\omega)$ are the normalized eigenfunctions of $\mathcal{L}^{2}$, with quantum numbers $q, \nu$. For each $q=0,1, \ldots$ there are $\nu_{q}$ values of $\nu$. Although the explicit formula for $\nu_{q}$ is rather clumsy, there is a simple bound for it, namely $\nu_{q} \leq C_{d} e^{\mu_{d} q}$. This bound suffices for the purpose of our proof. $L_{k}^{j}(x)$ denotes the Laguerre polynomial. By Lemma 6.2 of [8], this polynomial satisfies $\left|L_{k}^{q+\frac{d}{2}-1}(x)\right| \leq \frac{x^{k}}{k !}$ for all $x>4 k+2 q+d$. Finally, by equating the expressions for the energy, we obtain $|\beta|=2 k+q$. 
Now $\Phi_{\beta}(y)$ is certain linear combination of $\Psi_{k, q, \nu}(r, \omega)$,

$$
\Phi_{\beta}(y)=\sum_{\substack{k, q, \nu: \\ 2 k+q=|\beta|}} c_{k, q, \nu} \Psi_{k, q, \nu}(r, \omega)
$$

with $\sum\left|c_{k, q, \nu}\right|^{2}=1$. From (4.4), it follows that

$$
\begin{aligned}
\| \frac{\delta^{|\alpha|}}{\alpha !} D^{\alpha} V(\zeta) x^{\alpha^{\prime}} & \left(1-\chi_{R}\right) \Phi_{\beta}(x) \| \\
& \leq D_{1}\left\|\sum_{\substack{k, q, \nu: \\
2 k+q=|\beta|}} c_{k, q, \nu} e^{2 \frac{\tau}{\omega_{0}} y^{2}} y^{\alpha^{\prime}}\left(1-\chi_{R}\right) \Psi_{k, q, \nu}(y)\right\| \\
& \leq D_{1} \sum_{\substack{k, q, \nu: \\
2 k+q=|\beta|}}\left|c_{k, q, \nu}\right|\left\|e^{2 \frac{\tau}{\omega_{0}} y^{2}} y^{\alpha^{\prime}}\left(1-\chi_{R}\right) \Psi_{k, q, \nu}(y)\right\| \\
& \leq D_{1}\left(\sum_{\substack{k, q, \nu: \\
2 k+q=|\beta|}}\left|c_{k, q, \nu}\right|^{2}\left(\sum_{\substack{k, q, \nu: \\
2 k+q=|\beta|}}^{\frac{1}{2}}\left\|e^{2 \frac{\tau}{\omega_{0}} y^{2}} y^{\alpha^{\prime}}\left(1-\chi_{R}\right) \Psi_{k, q, \nu}(y)\right\|^{2}\right)^{\frac{1}{2}}\right.
\end{aligned}
$$

where we have used the Minkowski inequality followed by the Hölder inequality, along with some notational abuse. Therefore,

$$
\begin{aligned}
\left\|\frac{\delta^{|\alpha|}}{\alpha !} D^{\alpha} V(\zeta) x^{\alpha^{\prime}}\left(1-\chi_{R}\right) \Phi_{\beta}(x)\right\|^{2} \\
\leq D_{1}^{2} \sum_{\substack{k, q, \nu: \\
2 k+q=|\beta|}}\left\|e^{2 \frac{\tau}{\omega_{0}} y^{2}} y^{\alpha^{\prime}}\left(1-\chi_{R}\right) \Psi_{k, q, \nu}(y)\right\|^{2} \\
\leq D_{1}^{2} \sum_{\substack{k, q, \nu: \\
2 k+q=|\beta|}} \frac{2 k ! A_{d-1}}{\Gamma\left(k+q+\frac{d}{2}\right)} \int_{R}^{\infty} e^{-\left(1-\frac{4 \tau}{\omega_{0}}\right) r^{2}} r^{2(|\alpha|-1+q)}\left|L_{k}^{q+\frac{d}{2}-1}\left(r^{2}\right)\right|^{2} r^{d-1} d r
\end{aligned}
$$


where $A_{d-1}$ is the area of the $(d-1)$ dimensional unit sphere. We also have used that $y^{2\left|\alpha^{\prime}\right|} \leq r^{2\left|\alpha^{\prime}\right|}=r^{2(|\alpha|-1)}$. Since $R \geq \sqrt{2|\alpha|+d}$, Lemma 6.2 of [8] applies so

$$
\begin{aligned}
& \left\|\frac{\delta^{|\alpha|}}{\alpha !} D^{\alpha} V(\zeta) x^{\alpha^{\prime}}\left(1-\chi_{R}\right) \Phi_{\beta}(x)\right\|^{2} \\
& \leq D_{1}^{2} \sum_{\substack{k, q, \nu: \\
2 k+q=|\beta|}} \frac{2 A_{d-1}}{k ! \Gamma\left(k+q+\frac{d}{2}\right)} \int_{R}^{\infty} e^{-\left(1-\frac{4 \tau}{\omega_{0}}\right) r^{2}} r^{2|\alpha|+2 q+4 k+d-3} d r \\
& =D_{1}^{2} \sum_{\substack{k, q, \nu: \\
2 k+q=|\beta|}} \frac{2 A_{d-1}}{k ! \Gamma\left(k+q+\frac{d}{2}\right)} \frac{\Gamma\left(|\alpha|+q+2 k+\frac{d}{2}-1\right)}{2\left(1-\frac{4 \tau}{\omega_{0}}\right)^{|\alpha|+q+2 k+\frac{d}{2}-1}} \\
& =D_{1}^{2} A_{d-1} \frac{\Gamma\left(|\alpha|+|\beta|+\frac{d}{2}-1\right)}{\left(1-\frac{4 \tau}{\omega_{0}}\right)^{|\alpha|+|\beta|+\frac{d}{2}-1}} \sum_{\substack{k, q: \\
2 k+q=|\beta|}} \frac{\nu_{q}}{k ! \Gamma\left(k+q+\frac{d}{2}\right)} \\
& =D_{1}^{2} A_{d-1} \frac{\Gamma\left(|\alpha|+|\beta|+\frac{d}{2}-1\right)}{\left(1-\frac{4 \tau}{\omega_{0}}\right)^{|\alpha|+|\beta|+\frac{d}{2}-1}} \sum_{k=0}^{\llbracket \frac{|\beta|}{2} \rrbracket} \frac{\nu_{|\beta|-2 k}}{k ! \Gamma\left(|\beta|-k+\frac{d}{2}\right)} \\
& \leq D_{1}^{2} A_{d-1} C_{d} e^{\mu_{d}|\beta|} \frac{\Gamma\left(|\alpha|+|\beta|+\frac{d}{2}-1\right)}{\left(1-\frac{4 \tau}{\omega_{0}}\right)^{|\alpha|+|\beta|+\frac{d}{2}-1}} \sum_{k=0}^{\llbracket \frac{|\beta|}{2} \rrbracket} \frac{e^{-2 \mu_{d} k}}{k ! \Gamma\left(|\beta|-k+\frac{d}{2}\right)} .
\end{aligned}
$$

For $|\beta| \geq 1,|\beta|-k+d / 2 \geq 1+d / 2 \geq 2$ for all $0 \leq k \leq \llbracket \frac{|\beta|}{2} \rrbracket$. Since $\Gamma(x)$ is an increasing function for $x \geq 2$, we have

$$
\sum_{k=0}^{\llbracket \frac{|\beta|}{2} \rrbracket} \frac{e^{-2 \mu_{d} k}}{k ! \Gamma\left(|\beta|-k-\frac{d}{2}\right)} \leq \sum_{k=0}^{\llbracket \frac{|\beta|}{2} \rrbracket} \frac{1}{k !(|\beta|-k) !} \leq \frac{1}{|\beta| !} \sum_{k=0}^{|\beta|}\left(\begin{array}{c}
|\beta| \\
k
\end{array}\right)=\frac{1}{|\beta| !} 2^{|\beta|}
$$

For $|\beta|=0$, the sum above is smaller than $2 / \sqrt{\pi}$. Therefore

$$
\sum_{k=0}^{\llbracket \frac{|\beta|}{2} \rrbracket} \frac{e^{-2 \mu_{d} k}}{k ! \Gamma\left(|\beta|-k-\frac{d}{2}\right)} \leq \frac{2}{\sqrt{\pi}|\beta| !} 2^{|\beta|}
$$

for all $|\beta| \geq 0$. Thus (4.5) becomes

$$
\left\|\frac{\delta^{|\alpha|}}{\alpha !} D^{\alpha} V(\zeta) x^{\alpha^{\prime}}\left(1-\chi_{R}\right) \Phi_{\beta}(x)\right\|^{2} \leq D_{2}^{2} 2^{|\beta|} e^{\mu_{d}|\beta|} \frac{\Gamma\left(|\alpha|+|\beta|+\frac{d}{2}-1\right)}{|\beta| !\left(1-\frac{4 \tau}{\omega_{0}}\right)^{|\alpha|+|\beta|+\frac{d}{2}-1}}
$$


with $D_{2}^{2}:=2 D_{1}^{2} A_{d-1} C_{d} \pi^{-\frac{1}{2}}$.

Now consider any $\varphi \in \operatorname{Ran}\left(P_{|\beta| \leq 3 n+a+1}\right)$ so $\varphi=\sum_{|\beta| \leq 3 n+a+1} c_{\beta} \Phi_{\beta}(x)$. Then the Hölder inequality implies that

$\left\|\frac{\delta^{|\alpha|}}{\alpha !} D^{\alpha} V(\zeta) x^{\alpha^{\prime}}\left(1-\chi_{R}\right) P_{|\beta| \leq a+3 n+1} \varphi\right\|^{2} \leq\|\varphi\|^{2} \sum_{|\beta| \leq 3 n+a+1}\left\|\frac{\delta^{|\alpha|}}{\alpha !} D^{\alpha} V(\zeta) x^{\alpha^{\prime}}\left(1-\chi_{R}\right) \Phi_{\beta}(x)\right\|^{2}$.

Therefore

$$
\begin{aligned}
\left\|\frac{\delta^{|\alpha|}}{\alpha !} D^{\alpha} V(\zeta) x^{\alpha^{\prime}}\left(1-\chi_{R}\right) P_{|\beta| \leq a+3 n+1}\right\|^{2} & \\
& \leq D_{2}^{2} \frac{2^{3 n+a+1} e^{\mu_{d}(3 n+a+1)}}{\left(1-\frac{4 \tau}{\omega_{0}}\right)^{3 n+|\alpha|+a+\frac{d}{2}}} \sum_{|\beta| \leq 3 n+a+1} \frac{\Gamma\left(|\alpha|+|\beta|+\frac{d}{2}-1\right)}{|\beta| !} \\
& \leq D_{2}^{2} \frac{2^{3 n+a+1} e^{\mu_{d}(3 n+a+1)}}{\left(1-\frac{4 \tau}{\omega_{0}}\right)^{3 n+|\alpha|+a+\frac{d}{2}}} \sum_{|\beta| \leq 3 n+a+1} \frac{(|\alpha|+|\beta|+\llbracket d / 2 \rrbracket-1) !}{|\beta| !}
\end{aligned}
$$

where we use that $0<\left(1-4 \tau / \omega_{0}\right)<1$. The terms under the summation sign are increasing in $|\beta|$. Also,

$$
\begin{aligned}
\sum_{|\beta| \leq 3 n+a+1} 1 & =\sum_{s=0}^{3 n+a+1} \#\{\beta:|\beta|=s\} \\
& =\sum_{s=0}^{3 n+a+1} \frac{(s+d-1) !}{s !(d-1) !} \\
& \leq \sum_{s=0}^{3 n+a+1} \frac{(s+d-1)^{d-1}}{(d-1) !} \\
& \leq \frac{(3 n+a+d)^{d-1}}{(d-1) !}(3 n+a+2),
\end{aligned}
$$

and moreover, $(3 n+2+a) /(3 n+1+a) \leq 2$. Thus,

$$
\begin{aligned}
& \left\|\frac{\delta^{|\alpha|}}{\alpha !} D^{\alpha} V(\zeta) x^{\alpha^{\prime}}\left(1-\chi_{R}\right) P_{|\beta| \leq a+3 n+1}\right\|^{2} \\
& \leq D_{2}^{2} \frac{2^{3 n+a+2} e^{\mu_{d}(3 n+a+1)}}{\left(1-\frac{4 \tau}{\omega_{0}}\right)^{3 n+|\alpha|+a+\frac{d}{2}}}(3 n+a+d)^{d-1} \frac{(3 n+|\alpha|+\llbracket d / 2 \rrbracket+a) !}{(3 n+a) !} .
\end{aligned}
$$


Now define $C_{8}:=D_{2} e^{-\mu_{d} / 2}\left(1-4 \tau / \omega_{0}\right)^{1-d / 4}$ and $C_{9}:=\left[2 e^{\mu_{d}} /\left(1-4 \tau / \omega_{0}\right)\right]^{1 / 2}$.

Remark The argument above, as given, does not consider the one-dimensional case. In that case, however, the proof simplifies considerably. Refer to [27] for a detailed discussion.

Proof of Theorem 4.1. Recall that we assume that $\delta \leq 1$. We already know, from Theorem 3.2, that $b \geq 1$. From the proof of Lemma 2.3, we also know that $\left\|x_{i} A_{e}\right\| \leq \gamma$. Now, from (4.2), it follows that

$$
\begin{aligned}
\left\|A_{e}^{-1} \xi_{N}(x)\right\| \leq & \hbar^{\frac{N}{2}} \sum_{n=0}^{N-1} \sum_{|\alpha|=N-n+2}\left\|\frac{D^{\alpha} V\left(\zeta_{n}\right)}{\alpha !}\left(1-\chi_{R}(x)\right) x^{\alpha} A_{e} \psi_{n}(x)\right\| \\
& +\hbar^{\frac{N}{2}} \sum_{n=0}^{N-1} \sum_{|\alpha|=N-n+2}\left\|\frac{D^{\alpha} V\left(\zeta_{n}\right)}{\alpha !} \chi_{R}(x) x^{\alpha} A_{e} \psi_{n}(x)\right\| \\
& +\sum_{n=N}^{2 N-2} \hbar^{\frac{n}{2}} \sum_{l=n-N+1}^{N-1}\left\|\mathcal{E}_{l} A_{e} \psi_{n-l}(x)\right\| \\
\leq & \hbar^{\frac{N}{2}} \sum_{n=0}^{N-1} \sum_{|\alpha|=N-n+2}\left\|\frac{D^{\alpha} V\left(\zeta_{n}\right)}{\alpha !}\left(1-\chi_{R}(x)\right) x^{\alpha^{\prime}} P_{|\beta| \leq 3 n+a+1}\right\|\left\|x_{i} A_{e}\right\|\left\|\psi_{n}(x)\right\| \\
& +\hbar^{\frac{N}{2}} \sum_{n=0}^{N-1} \sum_{|\alpha|=N-n+2}\left\|\frac{D^{\alpha} V\left(\zeta_{n}\right)}{\alpha !} \chi_{R}(x) x^{\alpha^{\prime}} P_{|\beta| \leq 3 n+a+1}\right\|\left\|x_{i} A_{e}\right\|\left\|\psi_{n}(x)\right\| \\
& +\sum_{n=N}^{2 N-2} \hbar^{\frac{n}{2}} \sum_{l=n-N+1}^{N-1}\left|\mathcal{E}_{l}\right|\left\|\psi_{n-l}(x)\right\|
\end{aligned}
$$

where we split $x^{\alpha}$ into $x^{\alpha^{\prime}} x_{i}$, which is possible for some coordinate $x_{i}$ because $|\alpha| \geq 2$. Then $\left|\alpha^{\prime}\right|=|\alpha|-1$. Let us estimate each term on the right hand side of (4.6) individually. Applying Lemma 4.1 and the estimates for $\left\|x_{\alpha} A_{e}\right\|$ and $\left\|\psi_{n}\right\|$, we obtain

$$
\begin{aligned}
1^{\text {st }} \text { term } \leq & \hbar^{\frac{N}{2}} \sum_{n=0}^{N-1} \sum_{|\alpha|=N+2-n} \delta^{|\alpha|} C_{8} C_{9}^{\frac{3 n+a+2}{2}}\left(1-\frac{4 \tau}{\omega_{0}}\right)^{-\frac{|\alpha|}{2}}(3 n+a+d)^{\frac{d-1}{2}} \\
& \times\left[\frac{(3 n+|\alpha|+\llbracket d / 2 \rrbracket+a) !}{(3 n+a) !}\right]^{\frac{1}{2}} \gamma \kappa^{3 n} b^{n+w}[(1+a+n) !]^{\frac{1}{2}}
\end{aligned}
$$




$$
\begin{aligned}
\leq & C_{8} \gamma \hbar^{\frac{N}{2}} b^{N+w} \delta^{-(N+2)} C_{9}^{\frac{3 N+a+d+1}{2}}\left(1-\frac{4 \tau}{\omega_{0}}\right)^{-\frac{N+2}{2}}(3 N+a+d-3)^{\frac{d-1}{2}} \\
& \times \sum_{n=0}^{N-1} \kappa^{3 n}\left[\frac{(2 n+N+\llbracket d / 2 \rrbracket+a+2) !(n+a+1) !}{(3 n+a) !}\right]^{\frac{1}{2}} \sum_{|\alpha|=N+2-n} 1 .
\end{aligned}
$$

From the proof of Lemma 2.2 , we know that $\sum_{|\alpha|=N+2-n} 1 \leq[(d-1) !]^{-1}(N+d+1)^{d-1}$. Let us define $A_{1}:=\gamma \delta^{-2} b^{w} C_{8} C_{9}^{(a+d+1) / 2}\left[(d-1) !\left(1-4 \tau / \omega_{0}\right)\right]^{-1}$ and $B_{1}:=\delta^{-1} C_{9}^{3 / 2} b\left(1-4 \tau / \omega_{0}\right)^{-1}$. Then

$$
\begin{aligned}
1^{\text {st }} \text { term } \leq & A_{1} B_{1}^{N} \hbar^{\frac{N}{2}}(N+d+1)^{d-1}(3 N+a+d-3)^{\frac{d-1}{2}} \\
& \times \sum_{n=0}^{N-1} \kappa^{3 n}\left[\frac{(2 n+N+\llbracket d / 2 \rrbracket+a+2) !(n+a+1) !}{(3 n+a) !}\right]^{\frac{1}{2}} .
\end{aligned}
$$

Note that $(2 n+N+\llbracket d / 2 \rrbracket+a+2) ! \leq(2 n+N+a+2) !(2 n+N+\llbracket d / 2 \rrbracket+a+2)^{\llbracket d / 2 \rrbracket}$. Then

$$
\begin{aligned}
1^{\text {st }} \text { term } \leq & A_{1} B_{1}^{N} \hbar^{\frac{N}{2}}(3 N+a+d-3)^{\frac{d-1}{2}}(N+d+1)^{d-1}(3 N+a+\llbracket d / 2 \rrbracket)^{\frac{\llbracket d / 2 \rrbracket}{2}} \\
& \times[(2+a+N) !]^{\frac{1}{2}} \sum_{n=0}^{N-1} \kappa^{3 n}\left[\frac{(2+a+N+2 n) !(1+a+n) !}{(a+3 n) !(2+a+N) !}\right]^{\frac{1}{2}} \\
\leq & A_{1} B_{1}^{N} \kappa^{3 N} \hbar^{\frac{N}{2}}(3 N+a+d-3)^{\frac{d-1}{2}}(N+d+1)^{d-1}(3 N+a+\llbracket d / 2 \rrbracket)^{\frac{\llbracket d / 2 \rrbracket}{2}} \\
& \times[(2+a+N) !]^{\frac{1}{2}} \max _{1 \leq l \leq N}\left[\frac{(3 N-3 l+a+1)(3 N-3 l+a+2)}{(N-l+a+2)}\right]^{\frac{1}{2}} \\
& \times \sum_{l=1}^{N} \kappa^{-\frac{5 l}{2}}\left[\frac{(2+a+3 N+2 l) !(2+a+N-l) !}{(2+a+3 N-3 l) !(2+a+N) !}\right]^{\frac{1}{2}} .
\end{aligned}
$$

The change of index $n \rightarrow l=N-n$ was performed in the last sumation above. Now we need to apply Lemma 2.5, statement 2, to obtain

$$
\begin{aligned}
1^{\text {st }} \text { term } \leq & C_{5} A_{1} B_{1}^{N} \kappa^{3 N} \hbar^{\frac{N}{2}}(3 N+a+d-3)^{\frac{d-1}{2}}(N+d+1)^{d-1}(3 N+a+\llbracket d / 2 \rrbracket)^{\frac{\llbracket d / 2 \rrbracket}{2}} \\
& \times[3(3 N+a+2)]^{\frac{1}{2}}[(2+a+N) !]^{\frac{1}{2}}
\end{aligned}
$$


Finally define $N_{1}$ as the smallest integer such that the inequality

$$
(3 N+a+\llbracket d / 2 \rrbracket)^{\frac{\llbracket d / 2 \rrbracket}{2}}(3 N+a+d-3)^{\frac{d-1}{2}}(N+d+1)^{d-1}[3(3 N+a+2)]^{\frac{1}{2}} \leq \kappa^{N}
$$

holds for all $N \geq N_{1}$. Then, whenever $N \geq N_{1}$,

$$
1^{\text {st }} \text { term } \leq C_{5} A_{1} B_{1}^{N} \kappa^{4 N} \hbar^{\frac{N}{2}}[(2+a+N) !]^{\frac{1}{2}} .
$$

Statement 2 of Lemma 2.2 yields

$$
\frac{\delta^{|\alpha|}}{\alpha !}\left|D^{\alpha} V(\zeta(x))\right| \leq C_{0} \exp \left(\frac{2 \tau d}{\omega_{0}^{2}} R^{2}\right)=C_{0} \exp \left[\frac{2 \tau d}{\omega_{0}^{2}}(2 a+d-4)\right] \exp \left(\frac{12 \tau d}{\omega_{0}^{2}} N\right)
$$

on the support of $\chi_{R}(x)$. Thus, the second term of (4.6) satisfies

$$
\begin{aligned}
2^{\text {nd }} \text { term } \leq & \hbar^{\frac{N}{2}} \gamma \delta^{-(N+2)} C_{0} \exp \left[\frac{2 \tau d}{\omega_{0}^{2}}(2 a+d-4)\right] \exp \left(\frac{12 \tau d}{\omega_{0}^{2}} N\right) \\
& \times \sum_{n=0}^{N-1} \sum_{|\alpha|=N-n+2}\left\|x^{\alpha^{\prime}} P_{|\beta| \leq 3 n+a+1}\right\|\left\|\psi_{n}(x)\right\| \\
\leq & \hbar^{\frac{N}{2}} \gamma \delta^{-(N+2)} C_{0} \exp \left[\frac{2 \tau d}{\omega_{0}^{2}}(2 a+d-4)\right] \exp \left(\frac{12 \tau d}{\omega_{0}^{2}} N\right) \\
& \times \sum_{n=0}^{N-1} \sum_{|\alpha|=N-n+2} \kappa^{\frac{|\alpha|-1}{2}}\left[\frac{(a+|\alpha|+3 n) !}{(1+a+3 n) !}\right]^{\frac{1}{2}} \kappa^{3 n} b^{n+w}[(1+a+n) !]^{\frac{1}{2}} \\
\leq & \hbar^{\frac{N}{2}} \gamma \delta^{-(N+2)} C_{0} \exp \left[\frac{2 \tau d}{\omega_{0}^{2}}(2 a+d-4)\right] \exp \left(\frac{12 \tau d}{\omega_{0}^{2}} N\right) b^{N+w} \kappa^{\frac{N+1}{2}} \\
& \times \sum_{n=0}^{N-1} \kappa^{\frac{5 n}{2}}\left[\frac{(2+a+N+2 n) !(1+a+n) !}{(1+a+3 n) !} \sum_{|\alpha|=N-n+2}^{\frac{1}{2}} 1 .\right.
\end{aligned}
$$

Define $A_{2}:=\gamma \delta^{-2} \kappa^{1 / 2} C_{0} b^{w} \exp [2 \tau d(2 a+d-4)][(d-1) !]^{-1}$ and $B_{2}:=\delta^{-1} \kappa^{1 / 2} b \exp \left(12 \tau d / \omega_{0}^{2}\right)$.

Then, following the argument we have used to estimate the first term, we obtain

$$
2^{\text {nd }} \text { term } \leq A_{2} B_{2}^{N} \hbar^{\frac{N}{2}}(N+d+1)^{d-1} \sum_{n=0}^{N-1} \kappa^{3 n}\left[\frac{(2+a+N+2 n) !(1+a+n) !}{(1+a+3 n) !}\right]^{\frac{1}{2}}
$$




$$
\begin{aligned}
\leq & A_{2} B_{2}^{N} \kappa^{3 N} \hbar^{\frac{N}{2}}(N+d+1)^{d-1}[(2+a+N) !]^{\frac{1}{2}} \\
& \times \max _{1 \leq l \leq N}\left[\frac{2+a+3 N-3 l}{2+a+N-l}\right]^{\frac{1}{2}} \sum_{l=1}^{N} \kappa^{-\frac{5 l}{2}}\left[\frac{(2+a+3 N+2 l) !(2+a+N-l) !}{(2+a+3 N-3 l) !(2+a+N) !}\right]^{\frac{1}{2}} \\
\leq & 3^{\frac{1}{2}} C_{5} A_{2} B_{2}^{N} \kappa^{3 N} \hbar^{\frac{N}{2}}(N+d+1)^{d-1}[(2+a+N) !]^{\frac{1}{2}} .
\end{aligned}
$$

Now define $N_{2}$ such that $(N+d+1)^{d-1} \leq \kappa^{N}$ for every $N \geq N_{2}$. Then

$$
2^{\text {nd }} \text { term } \leq 3^{\frac{1}{2}} C_{5} A_{2} B_{2}^{N} \kappa^{4 N} \hbar^{\frac{N}{2}}[(2+a+N) !]^{\frac{1}{2}} .
$$

For the third term of (4.6), we only need to use the first statement of Lemma 2.5. The result is

$$
3^{\text {rd }} \text { term } \leq \sum_{n=N}^{2 N} C_{4} \kappa^{3 n} b^{n+2 w} \hbar^{\frac{N}{2}}[(1+a+n) !]^{\frac{1}{2}}
$$

To complete the proof define $N_{0}=\max \left\{N_{1}, N_{2}\right\}, A=\max \left\{C_{5} A_{1}, 3^{\frac{1}{2}} C_{5} A_{2}, C_{4} b^{2 w}\right\}$ and $B=\max \left\{\kappa^{4} B_{1}, \kappa^{3} B_{2}, \kappa^{3} b\right\}$. 


\section{Chapter 5}

\section{Optimal truncation}

In this chapter we shall prove that exact eigenvalues and eigenfunctions of $H(\hbar):=-\frac{1}{2} \Delta_{x}+$ $V(\hbar, x)$ can be approximated by truncated R-S series, up to an exponentially small error. To that end, we shall use our estimate of the norm $A_{e}^{-1} \xi_{N}(x)$. We shall also need a couple of results. The first is a lower bound for the distance between perturbed eigenvalues that degenerate at $\hbar=0$. The second is a "reverse" definition of asymptoticness.

Let us consider two distinct eigenvalues of $H(\hbar), E(\hbar)$ and $E^{\prime}(\hbar)$, which converge to the same eigenvalue of $H_{0}$ as $\hbar$ goes to 0 . Also, let us assume that their asymptotic series have only a finite number of common R-S coefficients. That is,

$$
\begin{aligned}
& E(\hbar) \sim e+\mathcal{E}_{1} \hbar^{\frac{1}{2}}+\ldots+\mathcal{E}_{M-1} \hbar^{\frac{M-1}{2}}+\mathcal{E}_{M} \hbar^{\frac{M}{2}}+\mathcal{E}_{M+1} \hbar^{\frac{M+1}{2}}+\ldots \\
& E^{\prime}(\hbar) \sim e+\mathcal{E}_{1} \hbar^{\frac{1}{2}}+\ldots+\mathcal{E}_{M-1} \hbar^{\frac{M-1}{2}}+\mathcal{E}_{M}^{\prime} \hbar^{\frac{M}{2}}+\mathcal{E}_{M+1}^{\prime} \hbar^{\frac{M+1}{2}}+\ldots
\end{aligned}
$$


with $\mathcal{E}_{M} \neq \mathcal{E}_{M}^{\prime}$. Then,

$$
E(\hbar)-E^{\prime}(\hbar) \sim\left(\mathcal{E}_{M}-\mathcal{E}_{M}^{\prime}\right) \hbar^{\frac{M}{2}}+\left(\mathcal{E}_{M+1}-\mathcal{E}_{M+1}^{\prime}\right) \hbar^{\frac{M+1}{2}}+\ldots
$$

so we expect that the difference between these exact eigenvalues be bounded below by $O\left(\hbar^{M / 2}\right)$. Since the series above is asymptotic, there are $C_{M}>0$ and $\hbar_{a}(M)>0$ so that

$$
\left|E(\hbar)-E^{\prime}(\hbar)-\left(\mathcal{E}_{M}-\mathcal{E}_{M}^{\prime}\right) \hbar^{\frac{M}{2}}\right| \leq C_{M} \hbar^{\frac{M+1}{2}}
$$

whenever $\hbar \leq \hbar_{a}(M)$. Then

$$
\left|E(\hbar)-E^{\prime}(\hbar)\right| \geq\left|\mathcal{E}_{M}-\mathcal{E}_{M}^{\prime}\right| \hbar^{\frac{M}{2}}-C_{M} \hbar^{\frac{M+1}{2}}
$$

Set $\hbar_{b}(M)=\left|\mathcal{E}_{M}-\mathcal{E}_{M}^{\prime}\right| / 2 C_{M}$. Then for $\hbar \leq \hbar_{b}(M)$,

$$
C_{M} \hbar^{\frac{M+1}{2}} \leq \frac{1}{2}\left|\mathcal{E}_{M}-\mathcal{E}_{M}^{\prime}\right| \hbar^{\frac{M}{2}}
$$

Thus for $\hbar \leq \hbar_{1}:=\min \left\{\hbar_{a}(M), \hbar_{b}(M)\right\}$ we have

$$
\left|E(\hbar)-E^{\prime}(\hbar)\right| \geq \frac{1}{2}\left|\mathcal{E}_{M}-\mathcal{E}_{M}^{\prime}\right| \hbar^{\frac{M}{2}}
$$

Let us denote $\mathcal{E}_{M}-\mathcal{E}_{M}^{\prime}$ as $\Delta \mathcal{E}_{M}$. Therefore, so far we know that

Lemma 5.1 Let $E(\hbar)$ and $E^{\prime}(\hbar)$ be distinct eigenvalues of $H(\hbar)$, which degenerate at $\hbar=0$.

Then either

1. $\left|E(\hbar)-E^{\prime}(\hbar)\right| \leq O\left(\hbar^{\frac{N}{2}}\right)$ for all non-negative integers $N$, or 
2. there exists $M$ and $\hbar_{1}=\hbar_{1}(M)$ such that

$$
\left|E(\hbar)-E^{\prime}(\hbar)\right| \geq \frac{1}{2}\left|\Delta \mathcal{E}_{M}\right| \hbar^{\frac{M}{2}}
$$

whenever $\hbar \leq \hbar_{1}$.

Remark It is clear that Lemma 5.1 is also valid when several eigenvalues of $H(\hbar)$ converge to the same eigenvalue of $H_{0}$. As a shorthand, we will say that $E(\hbar)$ is quasi-degenerate if the condition 1 in the lemma above occurs.

Lemma 5.2 Suppose $\sum_{n=0} f_{n} \beta^{n}$ is asymptotic to $f(\beta)$ in the sense that given $N \geq N_{0} \geq M$, there exists $C_{N}$ and $\beta(N)$ such that for all $\beta \leq \beta(N)$

$$
\left|f(\beta)-\sum_{n=0}^{N-1} f_{n} \beta^{n}\right|<C_{N} \beta^{N} .
$$

Then given $\epsilon>0$, there exists $\beta(\epsilon)>0$, such that for each $\beta \leq \beta(\epsilon)$, there is an $N(\beta) \geq N_{0}$ (maybe equal to $\infty$ ), so that

$$
\left|f(\beta)-\sum_{n=0}^{N-1} f_{n} \beta^{n}\right| \leq \epsilon \beta^{M}
$$

whenever $N_{0} \leq N<N(\beta)$.

Proof. Fix $\epsilon>0$. Define $\beta_{1}\left(N_{0}\right)=\left(\epsilon C_{N_{0}}^{-1}\right)^{\frac{1}{N_{0}-M}}$. Then for $N>N_{0}$, recursively choose positive numbers $\beta_{1}(N)$ that satisfy

$$
\beta_{1}(N)<\min \left\{\left(\epsilon C_{N}^{-1}\right)^{\frac{1}{N-M}}, \beta_{1}(N-1)\right\}
$$


Then

$$
\left|f(\beta)-\sum_{n=0}^{N-1} f_{n} \beta^{n}\right| \leq\left(C_{N} \beta^{N-M}\right) \beta^{M} \leq\left(C_{N} \beta_{1}(N)^{N-M}\right) \beta^{M} \leq \epsilon \beta^{M}
$$

whenever $\beta<\beta_{1}(N)$.

Define $\beta(\epsilon)=\beta_{1}\left(N_{0}\right)$, and define

$$
N(\beta)=\left\{\begin{array}{ccc}
N+1 & \text { if } & \beta_{1}(N+1)<\beta \leq \beta_{1}(N) \\
\infty & \text { if } \quad & \beta<\beta_{1}(N) \text { for all } N
\end{array}\right.
$$

Then (5.1) holds whenever $N_{0} \leq N \leq N(\beta)$.

Let $\left\{e_{I}\right\}_{I=0}^{\infty}$ be an arrangement in increasing order of the eigenvalues of $H_{0}$, counting multiplicities. Theorem 1.1 of [24] states that given a non-negative integer $J$, we can choose $\hbar_{0}$ so that for each $\hbar \leq \hbar_{0}$ there are at least $J+K$ eigenvalues of $H(\hbar)$, counting multiplicities. Furthermore, each one of them converges to one of the first $J+K$ eigenvalues of $H_{0}$. In the following proposition, we study the behavior of truncations of the R-S series of $E^{J}(\hbar)$, the $J$-th eigenvalue of $H(\hbar)$. We set $K$ so that $e_{J+K}>e_{J}$.

Proposition 5.1 Let $E(\hbar)=E^{J}(\hbar)$ be a non-quasi-degenerate eigenvalue of $H(\hbar)$, which converges to $e=e_{J}$. Let $E_{N}(\hbar)$ be the associated $R$-S series, truncated at order $N$. Let $N_{0}$ be as defined in Theorem 4.1. Then there exists $\hbar_{e}>0$ and for each $\hbar \leq \hbar_{e}$ there is an $N_{e}(\hbar) \geq N_{0}$ such that

$$
\left|E_{N}(\hbar)-E(\hbar)\right| \leq \sum_{n=N}^{2 N} A B^{n} \hbar^{\frac{n}{2}}[(2+a+n) !]^{\frac{1}{2}}
$$

for all $N_{0} \leq N \leq N_{e}(\hbar)$ 
Proof. We shall consider the case where there exists another eigenvalue of $H(\hbar)$ that converges to $e$. The proof can be easily simplified to accomodate the opposite situation, which is studied in Proposition 3 of [27]. So said, let $E^{\prime}(\hbar)$ be another eigenvalue of $H(\hbar)$ converging to $e$ as $\hbar \searrow 0$. By Lemma 5.1 , there are $M$ and $\hbar_{1}$ so that $\left|E(\hbar)-E^{\prime}(\hbar)\right| \geq \frac{1}{2}\left|\Delta \mathcal{E}_{M}\right| \hbar^{\frac{M}{2}}$ for $\hbar \leq \hbar_{1}$. Without loss we may assume that $N_{0} \geq M$. To simplify the proof, we furthermore assume that no other eigenvalue of $H(\hbar)$ converges to $e$. Let $G_{e}$ be the eigenspace associated to $e$.

Now set $N_{1}(\hbar)$ as the largest $N \geq N_{0}$ such that

$$
\sum_{n=N_{1}(\hbar)}^{2 N_{1}(\hbar)} A B^{n} \hbar^{\frac{n-M}{2}}[(2+a+n) !]^{\frac{1}{2}} \leq \frac{1}{4}\left|\Delta \mathcal{E}_{M}\right|
$$

Then, from Theorem 4.1 it follows that

$$
\left\|\left[H(\hbar)-E_{N}(\hbar)\right] A_{e} \Psi_{N}(\hbar ; x)\right\| \leq \sum_{n=N}^{2 N} A B^{n} \hbar^{\frac{n}{2}}[(2+a+n) !]^{\frac{1}{2}} \leq \frac{1}{4}\left|\Delta \mathcal{E}_{M}\right| \hbar^{\frac{M}{2}}
$$

whenever $\hbar \leq \hbar_{0}:=\min \left\{1,\left|\Delta \mathcal{E}_{M}\right|^{-2 / M}\right\}$ and $N_{0} \leq N \leq N_{1}(\hbar)$. On the other hand, note that $\Psi_{N}=\psi_{0}+\varphi_{N}$, where $\varphi_{N}$ is orthogonal to $\psi_{0} \in G_{e}$ because of the normalization we chose for the correction terms $\psi_{n}$. Since $A_{e} \psi_{0}=\psi_{0}$, we conclude that $\left\|A_{k} \Psi_{N}(\hbar ; x)\right\| \geq 1$. So Theorem 4.1 implies that

$$
\left\|\left[H(\hbar)-E_{N}(\hbar)\right] A_{e} \Psi_{N}(\hbar ; x)\right\| \leq \sum_{n=N}^{2 N} A B^{n} \hbar^{\frac{n}{2}}[(2+a+n) !]^{\frac{1}{2}}\left\|A_{e} \Psi_{N}(\hbar ; x)\right\| .
$$

We may assume that $E_{N}(\hbar) \notin \sigma(H(\hbar))$, so $\left[H(\hbar)-E_{N}(\hbar)\right]$ is invertible. It follows that

$$
\left\{\sum_{n=N}^{2 N} A B^{n} \hbar^{\frac{n}{2}}[(2+a+n) !]^{\frac{1}{2}}\right\}^{-1} \leq\left\|\left[H(\hbar)-E_{N}(\hbar)\right]^{-1}\right\| .
$$


Because $H$ is selfadjoint, $\left\|(H-E)^{-1}\right\|=\operatorname{dist}\{E, \sigma(H)\}^{-1}$ by the spectral theorem. Thus,

$$
\operatorname{dist}\left\{E_{N}(\hbar), \sigma(H)\right\} \leq \sum_{n=N}^{2 N} A B^{n} \hbar^{\frac{n}{2}}[(2+a+n) !]^{\frac{1}{2}} \leq \frac{1}{4}\left|\Delta \mathcal{E}_{M}\right| \hbar^{\frac{M}{2}}
$$

for $\hbar \leq \hbar_{0}$ and $N_{0} \leq N \leq N_{1}(\hbar)$. Let $\Delta$ be the minimum non-zero distance between the first $J+K$ eigenvalues of $H_{0}$. Since $E_{I}(\hbar) \rightarrow e_{I}$, we can set $\hbar_{\Delta}>0$ so that for $0 \leq I \leq J+K,\left|E_{I}(\hbar)-e_{I}\right| \leq \frac{1}{4} \Delta$ if $\hbar \leq \hbar_{\Delta}$. That implies that, for $\hbar \leq \hbar_{\Delta}$ and $E^{\prime \prime}(\hbar) \in \sigma(H(\hbar)) \backslash\left\{E(\hbar), E^{\prime}(\hbar)\right\}$

$$
\left|E^{\#}(\hbar)-E^{\prime \prime}(\hbar)\right| \geq \frac{1}{2} \Delta
$$

where $E^{\#}$ denotes either $E$ or $E^{\prime}$. Now set $\hbar_{2}=\left(\Delta /\left|\Delta \mathcal{E}_{M}\right|\right)^{\frac{2}{M}}$. Then for $\hbar \leq \hbar_{2}$ we have $\frac{1}{2} \Delta \geq \frac{1}{2}\left|\Delta \mathcal{E}_{M}\right| \hbar^{\frac{M}{2}}$. As a consequence,

$$
\begin{aligned}
\left|E(\hbar)-E^{\prime \prime}(\hbar)\right| \geq \frac{1}{2}\left|\Delta \mathcal{E}_{M}\right| \hbar^{\frac{M}{2}} \\
\left|E(\hbar)-E^{\prime}(\hbar)\right| \geq \frac{1}{2}\left|\Delta \mathcal{E}_{M}\right| \hbar^{\frac{M}{2}}
\end{aligned}
$$

which ultimately implies that

$$
\operatorname{dist}\{E(\hbar), \sigma(H) \backslash E(\hbar)\} \geq \frac{1}{2}\left|\Delta \mathcal{E}_{M}\right| \hbar^{\frac{M}{2}}
$$

whenever $\hbar \leq \min \left\{\hbar_{0}, \hbar_{1}, \hbar_{\Delta}, \hbar_{2}\right\}$. Since $E_{N}(\hbar)$ is asymptotic to $E(\hbar)$, we may apply Lemma 5.2. Then there is $\hbar_{3}>0$ such that for each $\hbar \leq \hbar_{3}$ we can fix $N_{2}(\hbar) \geq N_{0}$ so that

$$
\left|E(\hbar)-E_{N}(\hbar)\right| \leq \frac{1}{4}\left|\Delta \mathcal{E}_{M}\right| \hbar^{\frac{M}{2}}
$$

for $N_{0} \leq N \leq N_{2}(\hbar)$ 
Now (5.4), (5.5) and the second inequality of (5.3) implies that

$$
\operatorname{dist}\left\{E_{N}(\hbar), \sigma(H)\right\}=\left|E(\hbar)-E_{N}(\hbar)\right|
$$

whenever $\hbar \leq \min \left\{\hbar_{0}, \hbar_{1}, \hbar_{2}, \hbar_{3}, \hbar_{\Delta}\right\}=: \hbar_{e}$ and $N_{0} \leq N \leq \min \left\{N_{1}(\hbar), N_{2}(\hbar)\right\}=: N_{e}(\hbar)$.

Remark The number $N_{e}(\hbar)$ defined in the proof must indeed be equal to $N_{1}(\hbar)$. For assume that $N_{e}(\hbar)<N_{1}(\hbar)$, and consider $N_{e}(\hbar) \leq N \leq N_{1}(\hbar)$. Then $E_{N}(\hbar)$ has to be near some eigenvalue $E^{\prime \prime}(\hbar)$ different to $E(\hbar)$. By reducing $\hbar, E_{N}(\hbar)$ approaches to $E(\hbar)$ while keeping itself close to $E^{\prime \prime}(\hbar)$, which leads to a contradiction.

Remark $N_{e}(\hbar)$ grows like $g / \hbar$, as one can see from the proof of Theorem 5.1 below.

The requirement of $E(\hbar)$ to be non-quasi-degenerate can be relaxed, and formulate the following weaker version of Proposition 5.1. The proof is a straighforward variation of it.

Proposition 5.2 Let $E(\hbar)=E^{J}(\hbar)$ be an eigenvalue of $H(\hbar)$, which converges to $e=$ $e_{J}$. Let $E_{N}(\hbar)$ be the associated $R$-S series, truncated at order $N$. Also let $E^{\#}(\hbar)$ be any eigenvalue of $H(\hbar)$ that satisfies the condition 1 of Lemma 5.1 (including $E(\hbar)$ itself.) Then there exists $\hbar_{e}>0$ so that for each $\hbar \leq \hbar_{e}$ there is an $N_{e}(\hbar) \geq N_{0}$ such that

$$
\left|E_{N}(\hbar)-E^{\#}(\hbar)\right| \leq \sum_{n=N}^{2 N} A B^{n} \hbar^{\frac{n}{2}}[(2+a+n) !]^{\frac{1}{2}}
$$

for all $\hbar \leq \hbar_{e}, N_{0} \leq N \leq N_{e}(\hbar)$, and $E^{\#}(\hbar)$.

In the following theorem we assume the hypotheses of Proposition 5.1. An analogous result follows from the hypotheses of Proposition 5.2. 
Theorem 5.1 Assume the hypotheses of Proposition 5.1. Then for each $0<g<B^{-2}$, there is $\hbar_{g}>0$ such that for each $\hbar \leq \hbar_{g}$ there exists $N(\hbar)$ such that

$$
\left|E_{N(\hbar)}(\hbar)-E(\hbar)\right| \leq \Lambda \exp \left(-\frac{\Gamma}{\hbar}\right)
$$

for some $\Lambda>0$ and $\Gamma>0$ independent of $\hbar$.

Proof. Fix $0<g<B^{-2}$. Then $0<B^{2} g<1$, consequently there is $\Omega>0$ such that $B^{2} g=\exp (-\Omega)$. Consider the function

$$
f(\hbar):=A g \exp \left(-\frac{\Omega(1+a)}{4}\right) \hbar^{-\frac{4+a+M}{2}} \exp \left(-\frac{\Omega g}{4 \hbar}\right)
$$

It is clear that $f(\hbar)>0$ on $(0, \infty)$, has a single maximum, and $f(\hbar) \rightarrow 0$ as $\hbar \rightarrow 0$ or $\hbar \rightarrow \infty$.

Now set

$$
\hbar_{4}=\sup \left\{\hbar: f(\hbar) \text { is increasing and } f(\hbar) \leq \frac{1}{4}\left|\Delta \mathcal{E}_{M}\right|\right\}
$$

then set

$$
\hat{\hbar}_{g}=\sup \left\{\hbar: \hbar \leq \min \left\{\hbar_{e}, \hbar_{4}\right\} \text { and } \llbracket \frac{g}{\hbar} \rrbracket \geq 2+a+2 N_{0}\right\}
$$

Now for $\hbar \leq \hat{\hbar}_{g}$ define $N(\hbar)$ by $2+a+2 N(\hbar)=\llbracket \frac{g}{\hbar} \rrbracket$. So defined, $N(\hbar) \geq N_{0}$. On the other hand, since we can assume $B \geq 1$ and $2+a+n \leq g / \hbar$ for $N(\hbar) \leq n \leq 2 N(\hbar)$ we have

$$
\begin{aligned}
\sum_{n=N(\hbar)}^{2 N(\hbar)} A B^{n} \hbar^{\frac{n}{2}}[(2+a+n) !]^{\frac{1}{2}} & \leq \sum_{n=N(\hbar)}^{2 N(\hbar)} A B^{n} \hbar^{\frac{n}{2}}(2+a+n)^{\frac{2+a+n}{2}} \\
& \leq A \hbar^{-\frac{2+a}{2}} \sum_{n=N(\hbar)}^{2 N(\hbar)}\left[B^{2} \hbar(2+a+n)\right]^{\frac{2+a+n}{2}} \\
& \leq A \hbar^{-\frac{2+a}{2}} \sum_{n=N(\hbar)}^{2 N(\hbar)}\left(B^{2} g\right)^{\frac{2+a+n}{2}}
\end{aligned}
$$


Now use that $B^{2} g=\exp (-\Omega)<1$ and the fact that $x^{n} \geq x^{n+1}$ if $x \leq 1$ to obtain

$$
\begin{aligned}
\sum_{n=N(\hbar)}^{2 N(\hbar)} A B^{n} \hbar^{\frac{n}{2}}[(2+a+n) !]^{\frac{1}{2}} & \leq A \hbar^{-\frac{2+a}{2}} \sum_{n=N(\hbar)}^{2 N(\hbar)} \exp \left\{-\frac{\Omega}{2}[2+a+N(\hbar)]\right\} \\
& =A \hbar^{-\frac{2+a}{2}} e^{-\frac{\Omega}{4}(2+a)}[1+N(\hbar)] \exp \left\{-\frac{\Omega}{4}[2+a+2 N(\hbar)]\right\} \\
& \leq A \hbar^{-\frac{2+a}{2}} e^{-\frac{\Omega}{4}(2+a)}[2+a+2 N(\hbar)] \exp \left[-\frac{\Omega}{4}\left(\frac{g}{\hbar}-1\right)\right] \\
& \leq A g e^{-\frac{\Omega}{4}(1+a)} \hbar^{-\frac{4+a+M}{2}} \exp \left(-\frac{\Omega g}{4 \hbar}\right) \hbar^{\frac{M}{2}} \\
& \leq f\left(\hbar_{4}\right) \hbar^{\frac{M}{2}} \\
& \leq \frac{1}{4}\left|\Delta \mathcal{E}_{M}\right| \hbar^{\frac{M}{2}}
\end{aligned}
$$

Thus, $N(\hbar) \leq N_{e}(\hbar)$. Therefore, Proposition 5.1 holds for $\hbar<\hat{\hbar}_{g}$, which along with (5.6) implies

$$
\left|E_{N(\hbar)}(\hbar)-E(\hbar)\right| \leq A g e^{-\frac{\Omega}{4}(1+a)} \hbar^{-\frac{4+a}{2}} \exp \left(-\frac{\Omega g}{4 \hbar}\right)
$$

for all $\hbar \leq \hat{\hbar}_{g}$. Finally, define

$$
\hbar_{g}=\max \left\{\hbar \leq \hat{\hbar}_{g}: \hbar^{-\frac{4+a}{2}} \exp \left(-\frac{\omega g}{8 \hbar}\right) \leq 1\right\}
$$

Then the assertion is true for all $\hbar \leq \hbar_{g}$ with $\Gamma:=\Omega g / 8$ and $\Lambda:=A g \exp (-\Omega(1+a) / 4)$.

Proposition 5.3 Let $E(\hbar)$ be a non-quasi-degenerate eigenvalue of $H(\hbar)$, with eigenspace $G_{E}$. Let $P_{E}$ be the (orthogonal) projector onto $G_{E}$. Let $\tilde{\Psi}_{N}(\hbar ; x)$ be the $N^{\text {th }}$ truncation of the $R$-S series (2.5). Let $\hbar_{e}$ and $N_{e}(\hbar)$ be defined as in Proposition 5.1. Then for each $\hbar \leq \hbar_{e}$ and $N_{0} \leq N \leq N_{e}(\hbar)$,

$$
\left\|\frac{\tilde{\Psi}_{N}(\hbar ; x)}{\left\|\tilde{\Psi}_{N}(\hbar ; x)\right\|}-\frac{P_{E} \tilde{\Psi}_{N}(\hbar ; x)}{\left\|P_{E} \tilde{\Psi}_{N}(\hbar ; x)\right\|}\right\| \leq 16\left|\Delta \mathcal{E}_{M}\right|^{-1} \sum_{n=N}^{2 N} A B^{n} \hbar^{\frac{n-M}{2}}[(2+a+n) !]^{\frac{1}{2}}
$$

for some $M \leq N_{0}$. 
Proof. Notice that (5.2) means that

$$
\left\|\left[H(\hbar)-E_{N}(\hbar)\right]\right\| \tilde{\Psi}_{N}(\hbar ; x)\left\|^{-1} \tilde{\Psi}_{N}(\hbar ; x)\right\| \leq \sum_{n=N}^{2 N} A B^{n} \hbar^{\frac{n}{2}}[(2+a+n) !]^{\frac{1}{2}} .
$$

On the other hand, we can write

$$
\left\|\tilde{\Psi}_{N}(\hbar ; x)\right\|^{-1} \tilde{\Psi}_{N}(\hbar ; x)=w_{N}\left\|P_{E} \tilde{\Psi}_{N}(\hbar ; x)\right\|^{-1} P_{E} \tilde{\Psi}_{N}(\hbar ; x)+\Omega_{N}(\hbar ; x)
$$

where $\Omega_{N}(\hbar ; x)$ is orthogonal to $G_{E}$, and $\left|w_{N}\right|^{2}+\left\|\Omega_{N}(\hbar ; x)\right\|^{2}=1$. Since these functions are defined up to a global phase, we can assume that indeed $0<w_{n} \leq 1$. Then the normalization condition implies

$$
\left\|\Omega_{N}(\hbar ; x)\right\| \geq\left\|\Omega_{N}(\hbar ; x)\right\|^{2}=1-\left|w_{N}\right|^{2}=\left(1+w_{N}\right)\left(1-w_{N}\right) \geq 1-w_{N} .
$$

So we have

$$
\|\| \tilde{\Psi}_{N}(\hbar ; x)\left\|^{-1} \tilde{\Psi}_{N}(\hbar ; x)-\right\| P_{E} \tilde{\Psi}_{N}(\hbar ; x)\left\|^{-1} P_{E} \tilde{\Psi}_{N}(\hbar ; x)\right\| \leq 2\left\|\Omega_{N}(\hbar ; x)\right\| .
$$

Since

$$
\begin{aligned}
& {\left[H(\hbar)-E_{N}(\hbar)\right] \Omega_{N}(\hbar ; x)} \\
& \qquad=\left[H(\hbar)-E_{N}(\hbar)\right] \frac{\tilde{\Psi}_{N}(\hbar ; x)}{\left\|\tilde{\Psi}_{N}(\hbar ; x)\right\|}-w_{N}\left[E(\hbar)-E_{N}(\hbar)\right] \frac{P_{E} \tilde{\Psi}_{N}(\hbar ; x)}{\left\|P_{E} \tilde{\Psi}_{N}(\hbar ; x)\right\|},
\end{aligned}
$$

it follows from Proposition 5.1 that

$$
\left\|\left[H(\hbar)-E_{N}(\hbar)\right] \Omega_{N}(\hbar ; x)\right\| \leq 2 \sum_{n=N}^{2 N} A B^{n} \hbar^{\frac{n}{2}}[(2+a+n) !]^{\frac{1}{2}}
$$

for $\hbar \leq \hbar_{e}$ and $N_{0} \leq N \leq N_{e}(\hbar)$. 
Recall that $E_{N}(\hbar) \notin \sigma(H(\hbar))$. From the fact that $\left[H(\hbar)-E_{N}(\hbar)\right] \Omega_{N}(\hbar ; x)$ is orthogonal to $G_{E}$, it follows that

$$
\left\|\Omega_{N}(\hbar ; x)\right\| \leq\left\|\left[H(\hbar)-E_{N}(\hbar)\right]_{\perp}^{-1}\right\|\left\|\left[H(\hbar)-E_{N}(\hbar)\right] \Omega_{N}(\hbar ; x)\right\|
$$

where $\left[H(\hbar)-E_{N}(\hbar)\right]_{\perp}$ is the restriction of $\left[H(\hbar)-E_{N}(\hbar)\right]$ to the subspace orthogonal to $G_{E}$. For simplicity, let us assume that there is only one distinct eigenvalue $E^{\prime}(\hbar)$ that converges to the same eigenvalue of $H_{0}$ as $E(\hbar)$. Since

$$
\operatorname{dist}\left\{E_{N}(\hbar), \sigma(H) \backslash E(\hbar)\right\} \geq \frac{1}{2} \operatorname{dist}\{E(\hbar), \sigma(H) \backslash E(\hbar)\}
$$

the spectral theorem along with (5.4) imply that

$$
\left\|\left[H(\hbar)-E_{N}(\hbar)\right]_{\perp}^{-1}\right\| \leq 4\left|\Delta \mathcal{E}_{M}\right|^{-1} \hbar^{-\frac{M}{2}}
$$

The assertion now follows from (5.8)-(5.11).

Remark The assumption of non-quasi-degeneracy of $E(\hbar)$ is critical, as one can see in the argument that leads to (5.11).

The last result of this chapter concerns the optimal truncation for the eigenfunctions of $H(\hbar)$. It follows from Proposition 5.3 in the same way as Theorem 5.1 does from Proposition 5.1:

Theorem 5.2 Fix $0 \leq g \leq B^{-2}$. Let $\Lambda$ and $\Gamma$ be defined as in Theorem 5.1. Then there exists $\hbar_{g}^{\prime}>0$ such that for each $\hbar \leq \hbar_{g}^{\prime}$ there is $N(\hbar)$ so that

$$
\left\|\frac{\tilde{\Psi}_{N(\hbar)}(\hbar ; x)}{\left\|\tilde{\Psi}_{N(\hbar)}(\hbar ; x)\right\|}-\frac{P_{E} \tilde{\Psi}_{N(\hbar)}(\hbar ; x)}{\left\|P_{E} \tilde{\Psi}_{N(\hbar)}(\hbar ; x)\right\|}\right\| \leq 16\left|\Delta \mathcal{E}_{M}\right| \Lambda \exp \left(-\frac{\Gamma}{\hbar}\right)
$$


Proof. Define

$$
\begin{aligned}
f^{\prime}(\hbar) & :=\operatorname{Ag} \exp \left(-\frac{\Omega(1+a)}{4}\right) \hbar^{-\frac{4+a+2 M}{2}} \exp \left(-\frac{\Omega g}{4 \hbar}\right) \\
\hbar_{4}^{\prime} & :=\sup \left\{\hbar: f^{\prime}(\hbar) \text { is increasing and } f(\hbar) \leq \frac{1}{4}\left|\Delta \mathcal{E}_{M}\right|\right\} \\
\hat{\hbar}_{g}^{\prime} & :=\sup \left\{\hbar: \hbar \leq \min \left\{\hbar_{e}, \hbar_{4}^{\prime}\right\} \text { and } \llbracket \frac{g}{\hbar} \rrbracket \geq 2+a+2 N_{0}\right\} . \\
\hbar_{g}^{\prime} & :=\max \left\{\hbar \leq \hat{\hbar}_{g}^{\prime}: \hbar^{-\frac{4+a+M}{2}} \exp \left(-\frac{\omega g}{8 \hbar}\right) \leq 1\right\} .
\end{aligned}
$$

Now proceed as in the proof of theorem 5.1. 


\section{Chapter 6}

\section{Conclusion}

We have constructed exponentially accurate asymptotics to the solutions of the time independent Schrödinger equation in the limit $\hbar \searrow 0$. We have based our construction upon the standard scheme of partitioning the hamiltonian operator into a harmonic oscillator piece plus a residue, and then using the conceptually simple, formal Rayleigh-Schrödinger perturbation theory. A certain number of conditions have been required to the potential energy. Most notably, the potential energy has been assumed to be analytic and to grow not faster than $\exp \left(c x^{2}\right)$. However, we have been able to handle the case where the harmonic oscillator part has $\mathbb{Z}$-dependent eigenfrequencies. As we have mentioned in the Introduction, this latter situation has been the main restriction to the application of other techniques, like quantization of the canonical perturbation theory.

We conclude this work with a brief discussion of two issues. One refers to the relaxation 
of hypotheses. The other concerns the application of the ideas developed here to the BornOppenheimer approximation.

\subsection{Relaxing the hypotheses}

A closer look at the computations in Chapters 3 and 4 reveals that Theorem 4.1 does not depend entirely on hypotheses H1-H3. Rather, we may consider the following weaker assumptions:

H1* Let $V(x)$ be a $C^{\infty}$ real function on $\mathbb{R}^{d}$ bounded from below.

$\mathbf{H 2}^{*} V(x)$ has a local minimum $V(0)=0$ at $x=0$.

H3$^{*}$ The local minimum at the origin is non-degenerate in the sense that

$$
\operatorname{Hess}_{V}(0)=\operatorname{diag}\left[\omega_{1}^{2}, \ldots, \omega_{d}^{2}\right]
$$

has only strictly positive eigenfrequecies $\omega_{1}, \ldots, \omega_{d}$.

Hypothesis $H 1^{*}$ ensures that the operator $H(\hbar)$ is essentially self-adjoint in $C_{0}^{\infty}\left(\mathbb{R}^{d}\right)[21$, Thm. X.28], for all $\hbar>0$. More general cases could be accommodated. For instance, one might consider $C^{\infty}$ real functions that are bounded below by $-C|x|^{2}$ at infinity, according to the Faris-Lavine theorem [21, Thm. X.38]. But the goal here is to emphasize that our construction and estimate of the R-S series can be done around any non-degenerate local minimum. As before, the choice of the local minimun to be at the origin is made only for a 
sake of simplicity. With the new hypotheses, the following results follow from the proofs of Theorem 4.1 and 5.1:

Corollary 6.1 Theorem 4.1 holds if $V(x)$ satisfies $\mathrm{H}^{*}, \mathrm{H}_{2}^{*}, \mathrm{H}^{3}, \mathrm{H}_{4}$ and H5. That is, there exist positive constants $A, B$ and $N_{0}$ such that

$$
\left\|\left[H(\hbar)-E_{N}(\hbar)\right] \tilde{\Psi}_{N}(\hbar ; x)\right\| \leq \sum_{n=N}^{2 N} A B^{N} \hbar^{\frac{N}{2}}[(2+a+n) !]^{\frac{1}{2}},
$$

whenever $N_{0} \leq N$ and $\hbar \leq 1$.

Corollary 6.2 For $0 \leq g \leq B^{-2}$ there are $\Lambda$, $\Gamma$ and $\hbar_{g}>0$ such that, for each $\hbar \leq \hbar_{g}$, there is $N(\hbar)$ so that

$$
\left\|\left[H(\hbar)-E_{N(\hbar)}(\hbar)\right] \tilde{\Psi}_{N(\hbar)}(\hbar ; x)\right\| \leq \Lambda \exp \left(-\frac{\Gamma}{\hbar}\right)
$$

In the jargon of semiclassical analysis, a pair $\left.\left\{E(\hbar), \tilde{\Psi}_{(} \hbar, x\right)\right\}$ that satisfies inequality $(6.2)$ is called an exponentially accurate quasimode. Although quasimodes may look like approximate solutions to the time-independent Schrödinger equation, they are not necessarily close to eigensolutions of $H(\hbar)$ (which may not even exist). That is, the physical interpretation of quasimodes depends on the particular problem.

As we have mentioned in Chapter 2, the hypotheses $\mathrm{H} 4$ and $\mathrm{H} 5$ are crucial for the construction developed here. However, the computations presented in Chapter 3 might be generalized to Gevrey class potentials. In that case, the R-S coefficients are expected to grow as $b^{n}(n !)^{1 / \rho}$ with $\rho<2$. 


\subsection{Asymptotics on the Born-Oppenheimer approxi-}

\section{mation}

The hamiltonian for a molecular system can be written typically as

$$
H(\epsilon)=-\epsilon^{4} \Delta_{X}+H_{e}(X)
$$

where $X \in \mathbb{R}^{3 \nu}$ represents the nuclear coordinates, $\epsilon^{4}$ is the electron-nucleus ratio, and $H_{e}(X)$ is a family of Schrödinger operators that depends parametrically on the nuclear coordinates. $H(\epsilon)$ acts on a dense domain of $L^{2}\left(d^{3 \mu} x\right) \otimes L^{2}\left(d^{3 \nu} X\right)$, the Hilbert space for a molecule with $\mu$ electrons and $\nu$ nuclei. In the time-independent Born-Oppenheimer approximation, one first looks for solutions of the electronic hamiltonian $H_{e}(X)$ for fixed values of the nuclear coordinates. This yields a family of electronic energy surfaces that effectively act as potential energies for the nuclei. Then one solves the problem for the nuclear hamiltonian. The justification for the validity of this method is based on the fact that the electrons move much faster than the nuclei because of the disparity between their masses. In this approach, the electronic problem is treated in the adiabatic approximation. Finally, since $\epsilon$ is small, one may deal with the nuclear problem using semiclassical methods.

It is well known that the adiabatic limit leads to exponentially accurate approximations in terms of the adiabatic parameter. See, for instance, [18]. One may try to combine adiabatic methods with the construction that we have developed in this work, in order to obtain exponentially accurate asymptotics for the time-independent Born-Oppenheimer 
problem. However, the adiabatic and semiclassical contributions are deeply intertwined in this problem, which makes it technically difficult to separate them. In that sense, a technique which seems to be suitable for this problem is the so-called "method of multiple scales". This method was already used by Hagedorn in his study of the high order corrections to the BornOppenheimer approximation [7]. These ideas are the basis of an ongoing research project, whose results we expect to obtain in a near future. 


\section{Appendix A}

\section{Simplifying $\xi_{N}(x)$}

Here we simplify the formula (4.1) by using the the set of equations (2.9).

$$
\begin{aligned}
\xi_{N} & =\left[H_{e}+A_{e} W A_{e}-\sum_{j=1}^{N-1} \hbar^{\frac{j}{2}} \mathcal{E}_{j} A_{e}^{2}\right] \sum_{m=0}^{N-1} \hbar^{\frac{m}{2}} \psi_{m} \\
& =\sum_{m=0}^{N-1} \hbar^{\frac{m}{2}} H_{e} \psi_{m}+\sum_{m=0}^{N-1} \hbar^{\frac{m}{2}} A_{e} W A_{e} \psi_{m}-\sum_{j=1}^{N-1} \sum_{m=0}^{N-1} \hbar^{\frac{j+m}{2}} \mathcal{E}_{j} A_{e}^{2} \psi_{m} .
\end{aligned}
$$

We use $A_{e} W A_{e}=\sum_{j=3}^{N+2} \hbar^{\frac{j-2}{2}} T^{(j)}+A_{e} W^{[N+2]} A_{e}$ and change the index by $j \rightarrow j-2$. Using $H_{e} \psi_{0}=0$, we then obtain

$$
\begin{aligned}
\xi_{N}= & \sum_{m=1}^{N-1} \hbar^{\frac{m}{2}} H_{e} \psi_{m}+\sum_{m=0}^{N-1} \sum_{j=1}^{N} \hbar^{\frac{m+j}{2}} T^{(j+2)} \psi_{m} \\
& +\sum_{m=0}^{N-1} \hbar^{\frac{m}{2}} A_{e} W^{[N+2]} A_{e} \psi_{m}-\sum_{m=0}^{N-1} \sum_{j=1}^{N-1} \hbar^{\frac{j+m}{2}} \mathcal{E}_{j} A_{e}^{2} \psi_{m} \\
= & \sum_{n=1}^{N-1} \hbar^{\frac{n}{2}} H_{e} \psi_{n}+\sum_{n=1}^{N-1} \hbar^{\frac{n}{2}} \sum_{j=1}^{n} T^{(j+2)} \psi_{n-j} \\
& +\sum_{n=N}^{2 N-1} \hbar^{\frac{n}{2}} \sum_{j=n-N+1}^{N} T^{(j+2)} \psi_{n-j}+\sum_{m=0}^{N-1} \hbar^{\frac{m}{2}} A_{e} W^{[N+2]} A_{e} \psi_{m}
\end{aligned}
$$




$$
-\sum_{n=1}^{N-1} \hbar^{\frac{n}{2}} \sum_{j=1}^{n} \mathcal{E}_{j} A_{e}^{2} \psi_{n-j}-\sum_{n=N}^{2 N-2} \hbar^{\frac{n}{2}} \sum_{j=n-N+1}^{N-1} \mathcal{E}_{j} A_{e}^{2} \psi_{n-j} .
$$

The first, second and fifth terms of last equation cancel because of (2.9). In the third term define $m=n-j$ and then $p=n-N$. This yields

$$
\begin{aligned}
\xi_{N}= & \sum_{n=N}^{2 N-1} \hbar^{\frac{n}{2}} \sum_{m=n-N}^{N-1} T^{(n-m+2)} \psi_{m} \\
& +\sum_{m=0}^{N-1} \hbar^{\frac{m}{2}} A_{e} W^{[N+2]} A_{e} \psi_{m}-\sum_{n=N}^{2 N-2} \hbar^{\frac{n}{2}} \sum_{j=n-N+1}^{N-1} \mathcal{E}_{j} A_{e}^{2} \psi_{n-j} \\
= & \sum_{p=0}^{N-1} \sum_{m=p}^{N-1} \hbar^{\frac{p+N}{2}} T^{(p+N-m+2)} \psi_{m} \\
& +\sum_{m=0}^{N-1} \hbar^{\frac{m}{2}} A_{e} W^{[N+2]} A_{e} \psi_{m}-\sum_{n=N}^{2 N-2} \hbar^{\frac{n}{2}} \sum_{j=n-N+1}^{N-1} \mathcal{E}_{j} A_{e}^{2} \psi_{n-j} \\
= & \sum_{m=0}^{N-1} \hbar^{\frac{m}{2}} \sum_{p=0}^{m} \hbar^{\frac{p+N-m}{2}} T^{(p+N-m+2)} \psi_{m} \\
& +\sum_{m=0}^{N-1} \hbar^{\frac{m}{2}} A_{e} W^{[N+2]} A_{e} \psi_{m}-\sum_{n=N}^{2 N-2} \hbar^{\frac{n}{2}} \sum_{j=n-N+1}^{N-1} \mathcal{E}_{j} A_{e}^{2} \psi_{n-j} \\
= & \sum_{m=0}^{N-1} \hbar^{\frac{m}{2}}\left[\sum_{i=2}^{m+2} \hbar^{\frac{i+N-m-2}{2}} T^{(i+N-m)}+A_{e} W^{[N+2]} A_{e}\right] \psi_{m} \\
& -\sum_{n=N}^{2 N-2} \hbar^{\frac{n}{2}} \sum_{j=n-N+1}^{N-1} \mathcal{E}_{j} A_{e}^{2} \psi_{n-j} .
\end{aligned}
$$

Finally, note that $\hbar^{\frac{j-2}{2}} T^{(j)}+A_{e} W^{[j+1]} A_{e}=A_{e} W^{[j]} A_{e}$. Therefore, it follows that

$$
\xi_{N}=\sum_{m=0}^{N-1} \hbar^{\frac{m}{2}} A_{e} W^{[N-m+1]} A_{e} \psi_{m}-\sum_{n=N}^{2 N-2} \hbar^{\frac{n}{2}} \sum_{j=n-N+1}^{N-1} \mathcal{E}_{j} A_{e}^{2} \psi_{m-j} .
$$




\section{Bibliography}

[1] V. I. Arnold, Mathematical Methods of Classical Mechanics, Springer-Verlag, New York, 1989.

[2] D. Bambusi, S. Graffi and T. Paul, Normal forms and quantization formulae, Commun. Math. Phys. 207 (1999), no. 1, 173-195.

[3] J. M. Combes, P. Duclos and R. Seiler, Krein's formula and one-dimensional multiplewell, J. Funct. Anal. 52 (1983), no. 2, 257-301.

[4] J. M. Combes, P. Duclos and R. Seiler, Convergent expansion for tunneling, Commun. Math. Phys. 92 (1983), no. 2, 229-245.

[5] G. Gallavotti, The Elements of Mechanics, Springer-Verlag, New York, 1983.

[6] A. Grigis and J. Sjöstrand, Microlocal Analysis for Differential Operators: An Introduction, London Mathematical Series Lecture Note Series, 196, Cambridge University Press, 1994. 
[7] G. A. Hagedorn, High order corrections to the time-independent Born-Oppenheimer approximation. I. Smooth potentials, Ann. Inst. H. Poincaré Phys. Théor. 47 (1987), no. $1,1-16$.

[8] G. A. Hagedorn and A. Joye, Exponentially accurate semiclassical dynamics: propagation, localization, Ehrenfest times, scattering, and more general states, Ann. Henri Poincaré 1 (2000), no. 5, 837-883.

[9] B. Helffer, Semi-classical Analysis for the Schrödinger Operator and Applications, Lectures Notes in Mathematics, 1336, Springer-Verlag, Berlin, 1988.

[10] B. Helffer and J. Sjöstrand, Multiple wells in the semiclassical limit. I, Commun. Partial Differential Equations 9 (1984), no. 4, 337-408.

[11] J. S. Howland, Quasiclassical eigenvalues, J. Phys. A: Math. Gen. 30 (1997), no. 6, 2069-2076.

[12] W. Hunziker and C. A. Pillet, Degenerate asymptotic perturbation theory, Commun. Math. Phys. 90 (1983), no. 2, 219-233.

[13] W. Hunziker, Notes on asymptotic perturbation theory for Schrdinger eigenvalue problems, Helv. Phys. Acta 61 (1988), no. 3, 257-304.

[14] T. Kato, Perturbation Theory for Linear Operators, Springer-Verlag, Berlin, 1995.

[15] V. F. Lazutkin, KAM Theory and Semiclassical Approximations to Eigenfunctions, Springer-Verlag, Berlin, 1993. 
[16] A. Martinez, An Introduction to Semiclassical and Microlocal Analysis, Springer-Verlag, New York, 2002.

[17] A. Messiah, Quantum Mechanics, vol II, John Wiley \& Sons, Inc., New York, 1962.

[18] G. Nenciu, Linear adiabatic theory. Exponential estimates, Commun. Math. Phys. 152 (1993), no. 3, 479-496.

[19] G. Popov, Invariant tori, effective stability, and quasimodes with exponentially small error terms I - Birkhoff normal forms, Ann. Henri Poincaré 1 (2000), no. 2, 223-248.

[20] G. Popov, Invariant tori, effective stability, and quasimodes with exponentially small error terms II - quantum Birkhoff normal forms, Ann. Henri Poincaré 1 (2000), no. 2, 249-279.

[21] M. Reed and B. Simon, Methods of Modern Mathematical Physics, vol II, Academic Press, New York, 1975.

[22] M. Reed and B. Simon, Methods of Modern Mathematical Physics, vol III, Academic Press, New York, 1979.

[23] M. Reed and B. Simon, Methods of Modern Mathematical Physics, vol IV, Academic Press, New York, 1978.

[24] B. Simon, Semiclassical analysis of low lying eigenvalues. I. Nondegenerate minima: asymptotic expansions, Ann. Inst. Henri Poincaré A 38 (1983), no. 3, 295-308. 
[25] B. Simon, Semiclassical analysis of low lying eigenvalues. II. Tunneling, Ann. of Math. (2) 120 (1984), no. 1, 89-118.

[26] J. Sjöstrand, Semi-excited states in nondegenerate potential wells, Asymptotic Analysis 6 (1992), no. 1, 29-43.

[27] J. H. Toloza, Exponentially accurate error estimates of quasiclassical eigenvalues, J. Phys. A: Math. Gen. 34 (2001), no. 6, 1203-1218.

[28] J. H. Toloza, Exponentially accurate error estimates of quasiclassical eigenvalues II: several dimensions, preprint mp-arc 01-397.

[29] J. H. Toloza, Exponentially accurate semiclassical asymptotics, "Mathematical Results in Quantum Mechanics", R. Weder, P. Exner, B. Grebert (Eds.), AMS Contemporary Mathematics Series, CONM/307, 2002. 


\section{Vita}

Julio Hugo Toloza was born on August 23, 1970 in Córdoba, Argentina. After begining studies in an Electrical Engineering program, he changed fields to Physics at the National University of Córdoba in March 1991. He graduated in December 1996.

He won a Research Fellowship granted by the Research Council of the Province of Córdoba in April 1997. His first research experience was in Theoretical Condensed Matter.

In April 1998 he married Natacha Verónica Osenda.

In August 1998 he began graduate studies at Virginia Tech, and became a doctoral student in the Mathematical Physics program. Under the direction of Dr. George Hagedorn, he was awarded his doctorate in December 2002.

He expects that his academic career will continue as a full-time professor at the Institute of Sciences and Engineering of the University of State of Hidalgo, México. 